WHC-SP-1120

Revision 1

UC-900

\title{
Contracts and Management Services FY 1996 Site Support Program Plan WBS 6.10.14
}

J. M. Knoll, Jr.

Date Published

September 1995

Prepared for the U.S. Department of Energy

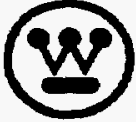

Management and Operations Contractor for the 


\section{DISCLAIMER}

Portions of this document may be illegible in electronic image products. Images are produced from the best available original document. 


\section{DISCLAIMER}

This report was prepared as an account of work sponsored by an agency of the United States Government. Neither the United States Government nor any agency thereof, nor any of their employees, make any warranty, express or implied, or assumes any legal liability or responsibility for the accuracy, completeness, or usefulness of any information, apparatus, product, or process disclosed, or represents that its use would not infringe privately owned rights. Reference herein to any specific commercial product, process, or service by trade name, trademark, manufacturer, or otherwise does not necessarily constitute or imply its endorsement, recommendation, or favoring by the United States Government or any agency thereof. The views and opinions of authors expressed herein do not necessarily state or reflect those of the United States Government or any agency thereof. 


\title{
Contracts and Management Services
}

\section{FY 1996 Site Support Program Plan}

\author{
WBS 6.10 .14
}

\section{WHC-SP-1120, Rev. 1}

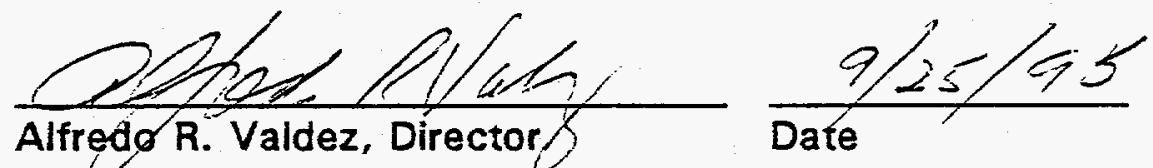

Procurement Services Division

U.S. Department of Energy

Richland Operations Office

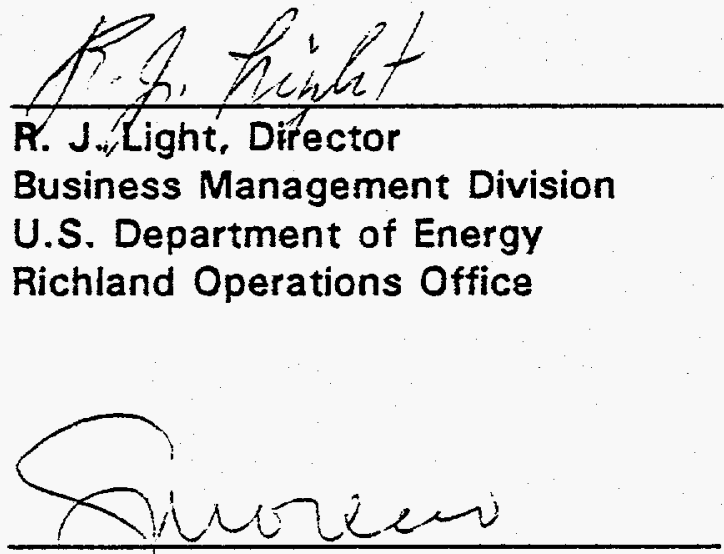

S. R.-Moreno

Office of General Counsel

Westinghouse Hanford Company
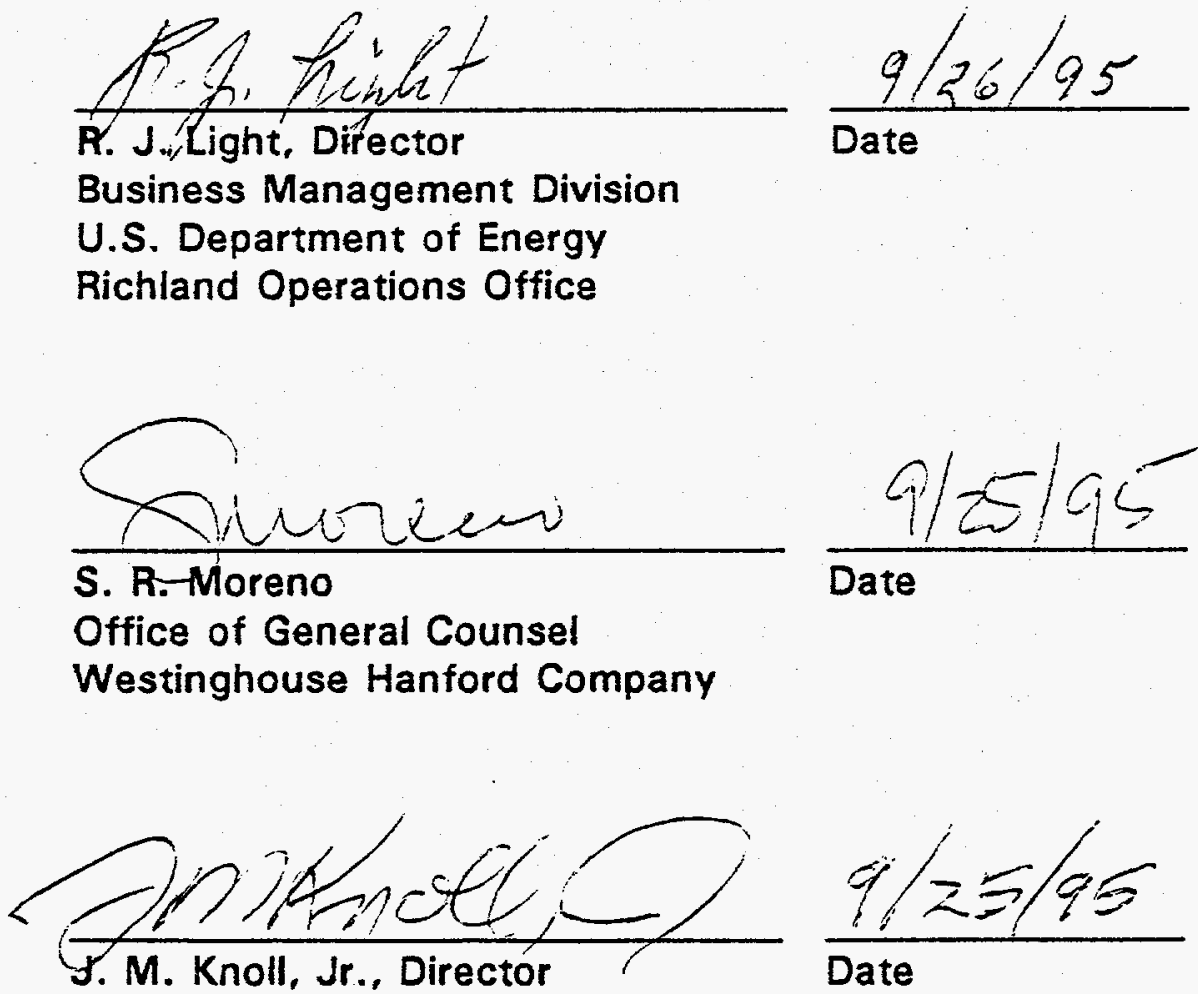

Contracts and Management Services

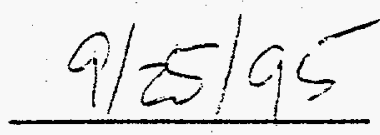

Office of General Counsel

Westinghouse Hanford Company

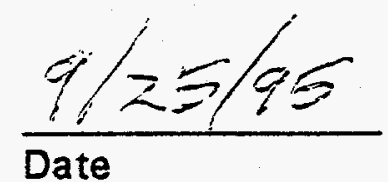




\section{RELEASE AUTHORIZATION}

Document Number: WHC-SP-1120, Rev. 1

Document Title: $\quad$ Contracts and Management Services FY 1996 Site Support Program Plan WBS 6.10.14

Release Date: $\quad 9 / 20 / 95$

This document was reviewed following the procedures described in WHC-CM-3-4 and is:

APPROVED FOR PUBLIC RELEASE

WHC Information Release Administration Specialist:

$$
\begin{aligned}
& \text { Chice Mrethigheso } \\
& \text { c. Willingham }
\end{aligned}
$$




\section{Program Mission}

Contracts and Management Services will support the Hanford mission financial and operational objectives by providing a central focal point for activities having company or sitewide application. Our aim is to be highly valued by our customers as a technically sound, cost effective, and safe operation. Our work will foster an atmosphere of ownership and teamwork, striving for continued improvement. and customer satisfaction. Contracts and Management Services will positively influence performance through integrating contract administration, major subcontract administration, company requirements, managing contract data and selected sitewide systems, and documenting cost reduction activity. 


\section{Program Objectives}

\section{Reengineering}

- Continue Reengineering Project Teams to identify reengineering opportunities for implementation.

- Retain CSC Index to provide expert counsel and guidance for the Reengineering effort.

- Develop Reengineering savings baseline and tracking approach. Savings achieved through Reengineering will be tracked in the Scorecard.

\section{Contracts Administration}

- Develop prime contract and subcontractor performance evaluation plans (PEP) to advance Contract Reform and improve the effectiveness of performance based incentives.

- Develop subcontract terms and conditions to alter subcontractor employee behavior leading to reduced costs, more tangible progress and full compliance with contract requirements.

- Design new or improved prime and subcontract performance based incentives which will result in lower cost and additional tangible production.

- Provide personal and organization incentives to reward behaviors leading to higher output performance.

- Complete subcontract evaluations on a timely basis.

- Promote completion of the EMSL project and other PNL projects on time and within budget.

- Complete transition and execute a memorandum of understanding between WHC and a possible new Hanford Site Integrator before the end of FY 1996, if needed.

- Closeout the prime contract, if needed.

- Install a streamlined cost based incentive to simplify the Challenge 170 program.

- Constructively improve the subjective award fee process on the prime contract and support RL's efforts to replace it with a more effective objective award fee process. 


\title{
Program Objectives (cont'd)
}

\author{
Contracts Administration (cont'd)
}

- Develop new sources for Hanford work including the spin-off of current scopes of work into employee-owned new businesses.

- Develop innovative acquisitions strategies and financing techniques for future construction projects on Site.

\section{Cost Management Program}

The strategy of the Cost Management Program, in accordance with DOE's Hanford Cost Savings Plan, is to improve cost effectiveness and management by implementing a coordinated set of systems to report company-wide process improvement initiatives.

- Develop and maintain the WHC cost Savings Plan in accordance with RL direction.

- Deveiop, implement, and maintain the Baseline Scorecard System, which includes tracking and reporting of the baseline and changes to the baseline, savings and funding. Ensure consistency and integrity of WHC data. Integrate other contractor data into the Scorecard.

- Administer the contractually-required cost incentive process for WHC. Coordinate ICF KH and BCSR cost incentive programs. Ensure claimed savings meet established savings criteria.

- Develop and maintain cost savings initiative descriptions and success stories; publicize successes.

\section{Contract Requirements Integration}

- Provide a leaner, more cost-effective system by eliminating extraneous, irrelevant material from documents.

- Eliminate redundant, inappropriate, or obsolete company-level documents.

- Shorten the cycle time required to issue procedures.

- Develop and submit Site Management System report for CMS.

- Develop Site Support Program Plan for CMS.

- Administer Liability Management Program. 


\section{Program Objectives (cont'd)}

\section{Contract Requirements Integration (cont'd)}

- Conduct process for requests for waivers to DOE Directives.

- Conduct process to establish WHC performance reports and the Self-Assessment Award Fee Report.

\section{Contract Data Management and Integration}

- To eliminate existing problems and/or avoid future problems, add at least 25 proposed data standards to the Data Standards Library during FY 1996.

- Deliver implementation plans to reduce or eliminate redundant computer system applications for at least six topical areas.

- Issue updated data management $p l$ ans to help identify redundancies and inefficiencies, and establish more cost-effective data management practices.

- Direct WHC's implementation of cost-effective and efficient data management practices with full and effective coordination with the Boeing Chief Information officer.

- Direct operations, applications, and interfaces contained within the Hanford PeopleCORE and Hanford Action Tracking Systems.

- Direct accomplishment and delivery of TPA data management milestones.

- Direct the sitewide processes for Standards and the Hanford Information System Inventory.

- Ensure corrective actions are tracked and reported within WHC/BCSR/ICF KH and RL in an efficient, timely manner.

G\&A Fee 


\section{Planning Assumptions}

\section{Reengineering}

- Senior Management-directed Reengineering Project Teams will continue to identify reengineering opportunities for implementation.

- Retain CSC Index to provide expert counsel and guidance for the reengineering effort.

- WHC Management is committed to reengineering as a significant tool in its cost savings plan.

\section{Contracts Administration}

- The current $M \& 0$ contract may be replaced with a site integrator contract on September 30, 1996.

- DOE HQ Contract Reform objectives will continue to evolve and the current prime contract will still be susceptible to Contract Reform-type improvements.

- DOE, in the interest of reducing costs, wil1 support using Hanford talent on off contract work with appropriate controls.

- DOE will continue to require outsourcing.

- DOE will evaluate private financing and will work with the financial community and WHC in an investment banking role.

- Prime Contract Performance Evaluation process will evolve.

\section{Cost Management Program}

- WHC will be incentivized to continue to reduce costs.

- WHC will support RL's efforts to transition to a process-oriented cost reduction approach.

- $\quad$ Provide company-wide cost savings tracking and reporting for use by WHC management and $\mathrm{RL}$ in reporting cost savings to $\mathrm{HQ}$ and regulators.

- Provide company-wide direction and training for Scorecard System and the Change Request data input. 


\section{Planning Assumptions (cont'd)}

\section{Contract Requirements Integration}

- Staffing projections do not include personnel for assuming responsibility for ICF Kaiser Hanford procedures.

- RL and TPA Milestone cost savings objectives remain the same.

Contract Data Management and Integration

- TPA data management requirements wi11 undergo minor changes.

- Data and information are primary deliverables in the Hanford mission. 


\section{Program Constraints}

\section{Reengineering}

None

\section{Contracts Administration}

- New performance based incentives continue to be developed and must be implemented without additional Contract Administration Staff.

- Contract Reform causes a continualiy changing contract environment.

- FY 1996 appears to be the final year for the M\&O contract and some added workload will be created for transitioning to successors and terminating subcontracts.

\section{Cost Management Program}

- Timeliness in completing negotiations of the FY 1996 cost incentive contract clause.

\section{Contract Requirements Integration}

- Integrated contractors establishing their own procedure systems for common use activities.

Contract Data Management and Integration

- Multiple changing contractor environment.

- $\quad$ Funding/Staffing unplanned changes. 


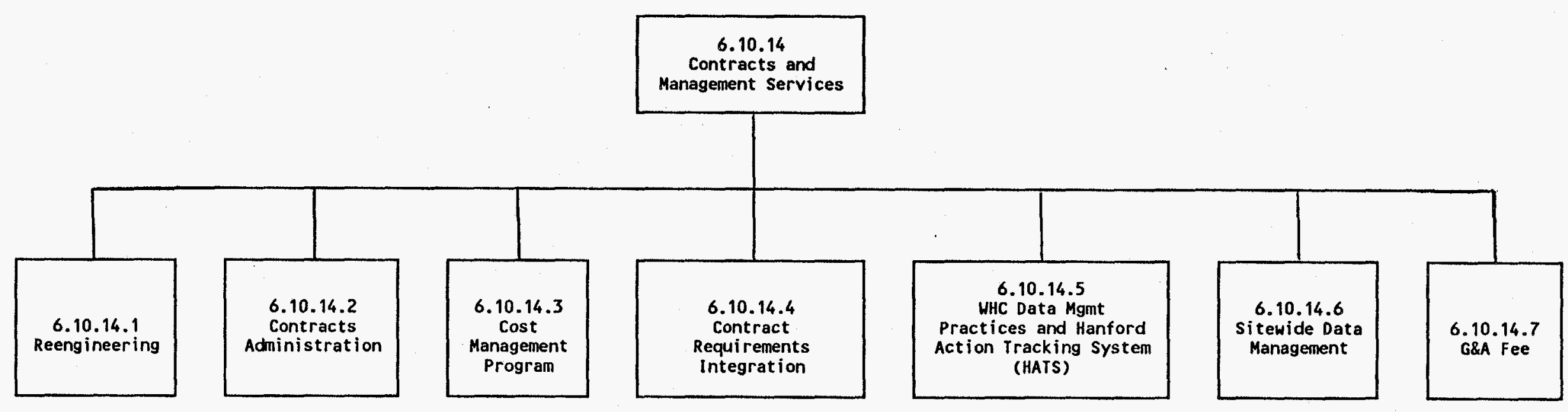




\begin{tabular}{|c|c|c|c|c|c|}
\hline \multicolumn{3}{|c|}{ York Breakdown. } & \multicolumn{2}{|c|}{ tructure Index and Programmatic Respons bl I lty } & \multirow{2}{*}{$\begin{array}{c}\text { Ass Ignment vatrix ix } \\
\begin{array}{c}\text { RESPONSIBLE } \\
\text { ORGANIZATION }\end{array} \\
\end{array}$} \\
\hline $\begin{array}{l}\text { PROGRAM } \\
\text { ELENENT } \\
\end{array}$ & ACTIVITY & $\begin{array}{c}\text { COST } \\
\text { ACCOUNT }\end{array}$ & TITLE & $\begin{array}{l}\text { RESPONSIBLE } \\
\text { MANAGER }\end{array}$ & \\
\hline 6.10 .14 & & & $\begin{array}{l}\text { Contracts \& Management } \\
\text { Services }\end{array}$ & J. M. Knoll, Jr. & $\begin{array}{l}\text { Contracts \& Management } \\
\text { Services }\end{array}$ \\
\hline & 6.10 .14 .1 & IMDDCS & Reengineering & J. R. Kirkendall & Cost Management Program Office \\
\hline & 6.10 .14 .2 & $1 M 0042$ & Contracts Administration & S. R. Morgan & Contracts Administration \\
\hline & 6.10 .14 .3 & 1MDD42 & Cost Management Program & J. R. Kirkendall & Cost Management Program Office \\
\hline & 6.10 .14 .4 & IMDD42 & $\begin{array}{l}\text { Contract Requirements } \\
\text { Integration }\end{array}$ & A. T. Broady & $\begin{array}{l}\text { Contract Requirements } \\
\text { Integration }\end{array}$ \\
\hline & 6.10 .14 .5 & $1 \mathrm{MDD} 42$ & $\begin{array}{l}\text { WHC Data Management } \\
\text { Practices and Hanford } \\
\text { Action Tracking System } \\
\text { (HATS) }\end{array}$ & M. K. Britton & $\begin{array}{l}\text { Contracts Data Management and } \\
\text { Integration }\end{array}$ \\
\hline & 6.10 .14 .6 & 1MDBPC & $\begin{array}{l}\text { Sitewide Data Management } \\
\text { (PeopleCORE, HISI, Data } \\
\text { Standards Process) } \\
\end{array}$ & M. K. Britton & $\begin{array}{l}\text { Contracts Data Management and } \\
\text { Integration }\end{array}$ \\
\hline & 6.10 .14 .7 & IMDDOA & G\&A Fee & S. R. Morgan & Contracts Administration \\
\hline & & & & & \\
\hline & & & & & - \\
\hline & & & & & \\
\hline & & & & & \\
\hline & & & & & \\
\hline & & & . & & \\
\hline
\end{tabular}




\begin{tabular}{|c|c|c|c|}
\hline \multicolumn{4}{|c|}{ MLESTONE I IST } \\
\hline $\begin{array}{l}\text { Milestone } \\
\text { Type }\end{array}$ & $\begin{array}{l}\text { Control } \\
\text { Number }\end{array}$ & Milestone Description & $\begin{array}{l}\text { Milestone } \\
\text { Completion Date }\end{array}$ \\
\hline $\mathrm{HQ} / \mathrm{RL}$ & CA-96-01 & Prime Contract Termination Proposal and Negotiations & $09 / 30 / 96$ \\
\hline RL & CA-96-02 & BCSR Subcontracts Termination Proposal and Negotiations & $09 / 30 / 96$ \\
\hline $\mathrm{RL}$ & CA-96-03 & ICF KH Subcontracts Termination Proposal and Negotiations & $09 / 30 / 96$ \\
\hline $\mathrm{RL}$ & CA-96-04 & BCSR Fiscal Year Performance Evaluation Plan & $09 / 01 / 96$ \\
\hline RL & CA-96-05 & ICF KH Fiscal Year Performance Evaluation Plan & $09 / 01 / 96$ \\
\hline RL & $\begin{array}{l}C A-96-06 A \\
C A-96-06 B \\
\end{array}$ & BCSR Six-Month Evaluation of Subcontracts & $\begin{array}{l}11 / 17 / 95 \\
05 / 17 / 96\end{array}$ \\
\hline RL & $\begin{array}{l}C A-96-07 A \\
C A-96-07 B \\
\end{array}$ & ICF KH Six-Month Evaluation of Subcontracts & $\begin{array}{l}11 / 17 / 95 \\
05 / 17 / 96\end{array}$ \\
\hline RL & $C A-96-08$ & Prime Contract Transition Plan & $06 / 30 / 96$ \\
\hline $\mathrm{RL}$ & CA-96-09 & Prime Contract Settlement Proposal & $09 / 30 / 96$ \\
\hline RL & CA-96-10 & $\begin{array}{l}\text { Creation of at Least One Employee-Owned or Locally-Owned Spin- } \\
\text { Off Performing Work Traditionally Performed In-House }\end{array}$ & $09 / 30 / 96$ \\
\hline RL & $\begin{array}{l}\text { CMP-96-01A } \\
\text { CMP-96-01B } \\
\text { CMP-96-01C } \\
\text { CMP-96-01D } \\
\text { CMP-96-01E } \\
\text { CMP-96-01F } \\
\text { CMP-96-01G } \\
\text { CMP-96-01H } \\
\text { CMP-96-01I } \\
\text { CMP-96-01J } \\
\text { CMP-96-01K } \\
\text { CMP-96-01L }\end{array}$ & Scorecard Report & $\begin{array}{c}11 / 30 / 95 \\
12 / 31 / 95 \\
\text { TBD } \\
\text { TBD } \\
\text { TBD } \\
\text { TBD } \\
\text { TBD } \\
\text { TBD } \\
\text { TBD } \\
\text { TBD } \\
\text { TBD } \\
\text { TBD } \\
\end{array}$ \\
\hline RL & CMP-96-02 & FY 1996 WHC Cost Savings Plan, Rev. 1, Submitted to RL & $10 / 31 / 95$ \\
\hline
\end{tabular}




\begin{tabular}{|c|c|c|c|}
\hline \multicolumn{4}{|c|}{ MLESTONE LIST } \\
\hline $\begin{array}{c}\text { Milestone } \\
\text { Type }\end{array}$ & $\begin{array}{l}\text { Control } \\
\text { Number }\end{array}$ & Milestone Description & $\begin{array}{c}\text { Milestone } \\
\text { Completion Date }\end{array}$ \\
\hline $\mathrm{RL}$ & CMP-96-03 & FY 1996 WHC Cost Savings Plan -- Final Submitted to RL & TBD \\
\hline RL & CMP-96-04 & FY 1996 WHC Cost Savings PIan - Other Revisions Submitted to RL & TBD \\
\hline $\mathrm{RL}$ & CMP-96-05 & FY 1995 Year-End Scorecard Submitted to RL & $11 / 10 / 95$ \\
\hline RL & CMP-96-06 & Support and Administration Reengineering Team Deliverables & TBD \\
\hline $\mathrm{RL}$ & CMP-96-07 & Plant Reengineering Team Deliverables & TBD \\
\hline $\mathrm{RL}$ & CMP-96-08 & Requirements Reengineering Team Deliverables & TBD \\
\hline RL & CMP-96-09 & Obtain R1 Approval of the Final FY 1996 Scorecard Format & $12 / 15 / 95$ \\
\hline $\mathrm{RL}$ & CMP-96-10 & Begin Submitting Scorecard to RL in New Format & $01 / 31 / 96$ \\
\hline $\mathrm{RL}$ & CMP-96-11 & Implement Electronically-Generated Scorecard (Not Funded) & $12 / 15 / 95$ \\
\hline $\mathrm{RL}$ & CMP-96-12 & Provide FY 1995 Year-End C-170 Fee Reconciliation to RL & $11 / 10 / 95$ \\
\hline
\end{tabular}

* TPA, HQ, RL, and selected contractor milestones

* On Tri-Party Agreement Milestones, also designate if they are $\mathrm{HQ}, \mathrm{RL}$ 


\begin{tabular}{|c|c|c|c|}
\hline \multicolumn{3}{|c|}{$\begin{array}{l}\text { Title: Prime Contract Termination Proposal } \\
\text { and Negotiations }\end{array}$} & $\begin{array}{l}\text { Date Prepared: } \\
08 / 28 / 95\end{array}$ \\
\hline \multicolumn{3}{|c|}{ Assigned To: Contracts Administration } & CIN: \\
\hline \multicolumn{3}{|c|}{ WBS Designator: 6.10 .14 .2} & Due Date: $9 / 30 / 96$ \\
\hline \multicolumn{3}{|c|}{ Control Number: CA-96-01 } & Revision: \\
\hline $\begin{array}{l}\text { Milestone Type: } \\
\text { DOE-HQ } \\
\text { DOE-RL } \\
\square \text { CNTR }\end{array}$ & $\begin{array}{ll} & \text { Division: } \\
\square & \text { State } \\
\square & \text { Federal } \\
\text { DOE } \\
\square \text { RCRA } \\
\square \text { TPA\# } \\
\end{array}$ & $\begin{array}{ll} & \text { DELIVERABLE: } \\
\square & \text { Report } \\
\square & \text { Letter } \\
\square & \text { Drawings } \\
\text { Other: Proposal } \\
\quad & \text { Package }\end{array}$ & $\begin{array}{l}\text { ADDRESS TO: } \\
\square \text { DOE-HQ } \\
\text { DOE-RL } \\
\square \text { other } \\
\text { (specify) }\end{array}$ \\
\hline
\end{tabular}

\section{Milestone Description:}

Prime Contract termination proposal and negotiations.

Description of what constitutes completion of this milestone:

Preparation and delivery of proposal to RL.

\begin{tabular}{|ll|lc|}
\hline $\begin{array}{l}\text { Cost Account Manager } \\
\text { J.M. Knoll, Jr. }\end{array}$ & Date & $\begin{array}{l}\text { Program/Project Manager } \\
\text { S. R. Morgan }\end{array}$ & Date \\
\hline $\begin{array}{l}\text { Program Element Manager } \\
\text { S. R. Morgan }\end{array}$ & Date & $\begin{array}{l}\text { DOE Monitor } \\
\text { A.W. Kellogg }\end{array}$ & Date \\
\hline
\end{tabular}



MILESTONE DESCRIPTION SHEET

\begin{tabular}{|c|c|c|c|}
\hline $\begin{aligned} & \text { Title: } \begin{array}{l}\text { BCSR Sul } \\
\text { and Neg }\end{array} \\
&\end{aligned}$ & $\begin{array}{l}\text { bcontracts Ter } \\
\text { otiations }\end{array}$ & ion Proposal & $\begin{array}{l}\text { Date Prepared: } \\
08 / 28 / 95\end{array}$ \\
\hline Assigned To: C & ontracts Admini & ition & CIN: \\
\hline WBS Designator: & 6.10 .14 .2 & & Due Date: $09 / 30 / 96$ \\
\hline Control Number: & CA-96-02 & & Revision: \\
\hline $\begin{array}{l}\text { Milestone } \\
\text { Type: } \\
\square \text { DOE-HQ } \\
\text { DOE-RL } \\
\square \text { CNTR }\end{array}$ & $\begin{array}{ll} & \text { Division: } \\
\square & \text { State } \\
\square & \text { Federa } \\
\text { DOE } \\
\square \\
\text { RCRA } \\
\square \text { TPA\# } \\
\end{array}$ & $\begin{array}{ll} & \text { DELIVERABLE: } \\
\square & \text { Report } \\
\square & \text { Letter } \\
\square & \text { Drawings } \\
\text { Other: Proposal } \\
\end{array}$ & $\begin{array}{l}\text { ADDRESS TO: } \\
\square \text { DOE-HQ } \\
\square \text { DOE-RL } \\
\text { Dther: BCSR }\end{array}$ \\
\hline
\end{tabular}

\section{Milestone Description:}

BCSR subcontracts termination proposal and negotiations.

Description of what constitutes completion of this milestone:

Receipt of and explanation of termination proposal from BCSR and completion of negotiations between BCSR and WHC.

\begin{tabular}{|ll|ll|}
\hline $\begin{array}{l}\text { Cost Account Manager } \\
\text { J.M. Kno17, Jr. }\end{array}$ & Date & $\begin{array}{l}\text { Program/Project Manager } \\
\text { S. R. Morgan }\end{array}$ & Date \\
\hline $\begin{array}{l}\text { Program Element Manager } \\
\text { S. R. Morgan }\end{array}$ & Date & $\begin{array}{l}\text { DOE Monitor } \\
\text { A. W. Kellogg }\end{array}$ & Date \\
\hline
\end{tabular}



MILESTONE DESCRIPTION SHEET

\begin{tabular}{|c|c|c|c|c|}
\hline \multicolumn{4}{|c|}{$\begin{array}{l}\text { Title: ICF KH Subcontracts Termination Proposal } \\
\text { and Negotiations }\end{array}$} & $\begin{array}{l}\text { Date Prepared: } \\
08 / 28 / 95\end{array}$ \\
\hline \multicolumn{4}{|c|}{ Assigned To: Contracts Administration } & CIN: \\
\hline \multicolumn{4}{|c|}{ WBS Designator: 6.10 .14 .2} & Due Date: $9 / 30 / 96$ \\
\hline \multicolumn{4}{|c|}{ Control Number: CA-96-03 } & Revision: \\
\hline $\begin{array}{l}\text { Milestone Type: } \\
\square \text { DOE-HQ } \\
\text { DOE-RL } \\
\square \text { CNTR }\end{array}$ & $\begin{array}{ll} & \text { Division: } \\
\square & \text { State } \\
\square & \text { Federal } \\
\text { DOE } \\
\square \text { RCRA } \\
\square \text { TPA\#\# } \\
\end{array}$ & $\begin{array}{l}\square \\
\square \\
\square\end{array}$ & $\begin{array}{l}\text { DELIVERABLE: } \\
\text { Report } \\
\text { Letter } \\
\text { Drawings } \\
\text { Other: Proposal } \\
\quad \begin{array}{l}\text { Package }\end{array}\end{array}$ & $\begin{array}{ll} & \text { ADDRESS TO: } \\
\square & \text { DOE-HQ } \\
\square & \text { DOE-RL } \\
\text { Other: ICF KH } & \text { Other }\end{array}$ \\
\hline
\end{tabular}

\section{Milestone Description:}

ICF KH Subcontracts termination proposal and negotiations.

Description of what constitutes completion of this milestone:

Receipt of and explanation of termination proposal from ICF KH, and completion of negotiations between ICF KH and WHC.

\begin{tabular}{|lc|lc|}
\hline $\begin{array}{l}\text { Cost Account Manager } \\
\text { J.M. Knoll, Jr. }\end{array}$ & Date & $\begin{array}{l}\text { Program/Project Manager } \\
\text { S. R. Morgan }\end{array}$ & Date \\
\hline $\begin{array}{l}\text { Program Element Manager } \\
\text { S. R. Morgan }\end{array}$ & Date & $\begin{array}{l}\text { DOE Monitor } \\
\text { A.W. Kellogg }\end{array}$ & Date \\
\hline
\end{tabular}




\begin{tabular}{|c|c|c|c|}
\hline Title: BCSR Fis & a] Year Perfo & Evaluation Plan & $\begin{array}{l}\text { Date Prepared: } \\
08 / 28 / 95\end{array}$ \\
\hline Assigned To: $\mathrm{Co}_{0}$ & tracts Adminis & & CIN: \\
\hline WBS Designator: & 6.10 .14 .2 & & Due Date: $09 / 01 / 96$ \\
\hline Control Number: & CA-96-04 & & Revision: \\
\hline $\begin{array}{l}\text { Milestone Type: } \\
\square \text { DOE-HQ } \\
\square \text { DOE-RL } \\
\square \text { CNTR }\end{array}$ & $\begin{array}{ll} & \text { Division: } \\
\square & \text { State } \\
\square & \text { Federal } \\
\text { DOE } \\
\square \text { RCRA } \\
\square \text { TPA\# }\end{array}$ & $\begin{array}{ll} & \text { DELIVERABLE: } \\
\square & \text { Report } \\
\square & \text { Letter } \\
\square & \text { Drawings } \\
& \text { Other: Plan }\end{array}$ & $\begin{array}{ll} & \text { ADDRESS TO: } \\
\square & \text { DOE-HQ } \\
\square & \text { DOE-RL } \\
\text { Other: BCSR }\end{array}$ \\
\hline
\end{tabular}

\section{Milestone Description:}

BCSR fiscal year evaluation plan as provided for in the BCSR subcontract WHC-50930.

\section{Description of what constitutes completion of this milestone:}

A Performance Evaluation Plan prepared and negotiated with BCSR and submitted to RL for approval. Completion occurs when submitted to RL.

\begin{tabular}{|lc|ll|}
\hline $\begin{array}{l}\text { Cost Account Manager } \\
\text { J.M. Knoll, Jr. }\end{array}$ & Date & $\begin{array}{l}\text { Program/Project Manager } \\
\text { S. R. Morgan }\end{array}$ & Date \\
\hline $\begin{array}{l}\text { Program Element Manager } \\
\text { S. R. Morgan }\end{array}$ & Date & $\begin{array}{l}\text { DOE Monitor } \\
\text { A. W. Kellogg }\end{array}$ & Date \\
\hline
\end{tabular}




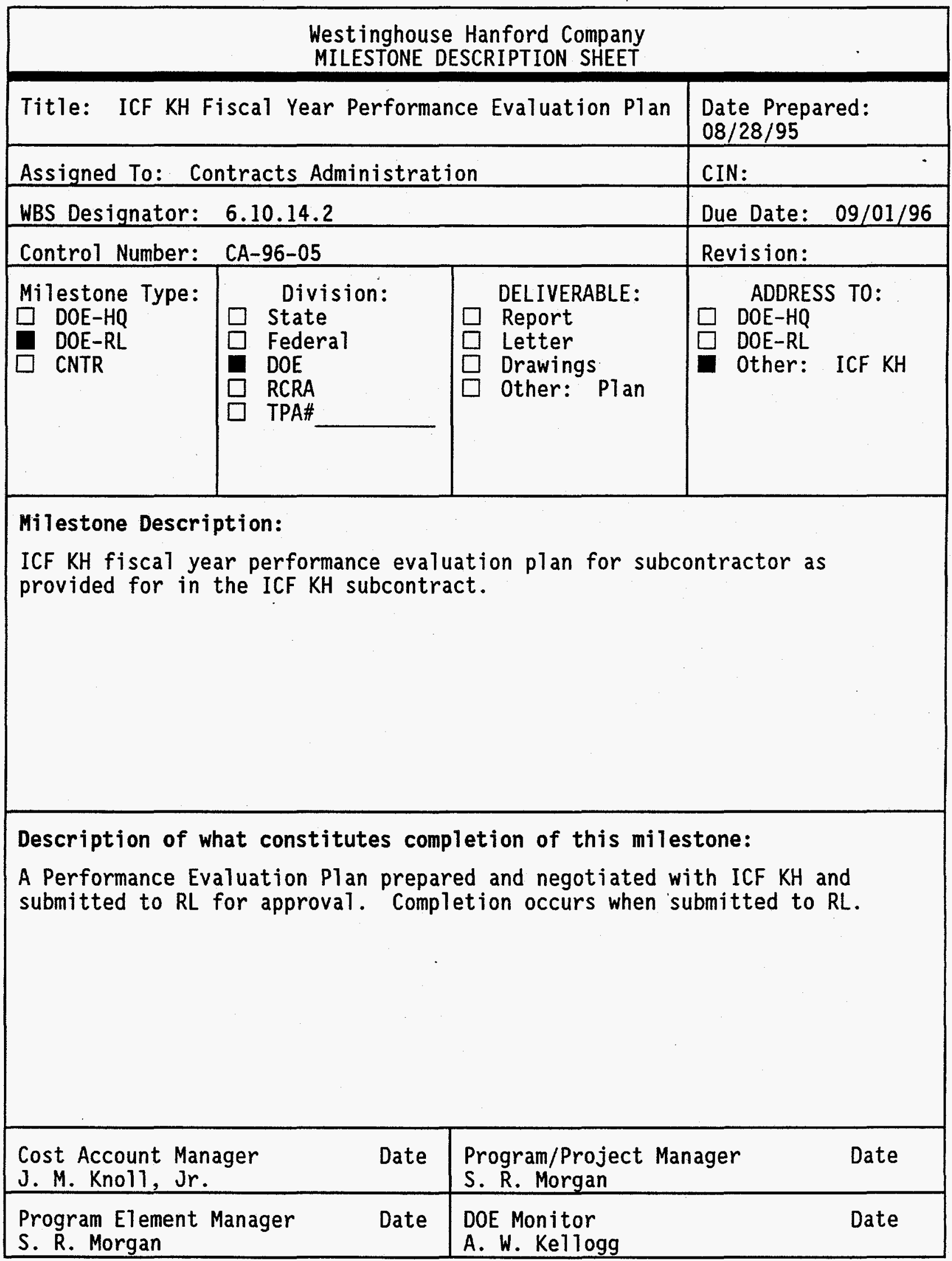


FY 1996 Program Plan

\section{CONTRACTS AND MANAGEMENT SERVICES}

WBS 6.10 .14

WHC-SP-1120

Westinghouse Hanford Company

MILESTONE DESCRIPTION SHEET

\begin{tabular}{|l|l|}
\hline Title: BCSR Six Month Evaluation of Subcontracts & $\begin{array}{l}\text { Date Prepared: } \\
08 / 28 / 95\end{array}$ \\
\hline Assigned To: Contracts Administration & CIN: \\
\hline WBS Designator: 6.10.14.2 & Due Date: 11/17/95 \\
$05 / 19 / 96$
\end{tabular}

\section{Milestone Description:}

BCSR six month evaluation of subcontract performance for fee determination.

Description of what constitutes completion of this milestone:

Completion occurs when the decisional is submitted to RL along with the request for written formal approval from $\mathrm{RL}$.

\begin{tabular}{|lc|lc|}
\hline $\begin{array}{l}\text { Cost Account Manager } \\
\text { J.M. Knoll, Jr. }\end{array}$ & Date & $\begin{array}{l}\text { Program/Project Manager } \\
\text { S. R. Morgan }\end{array}$ & Date \\
\hline $\begin{array}{l}\text { Program Element Manager } \\
\text { S. R. Morgan }\end{array}$ & Date & $\begin{array}{l}\text { DOE Monitor } \\
\text { A. W. Kellogg }\end{array}$ & Date \\
\hline
\end{tabular}


Westinghouse Hanford Company

MILESTONE DESCRIPTION SHEET

\begin{tabular}{|c|c|c|c|}
\hline \multicolumn{3}{|c|}{ Title: ICF KH Six Month Evaluation of Subcontracts } & $\begin{array}{l}\text { Date Prepared: } \\
08 / 28 / 95\end{array}$ \\
\hline \multicolumn{3}{|c|}{ Assigned To: Contracts Administration } & CIN: \\
\hline \multicolumn{3}{|c|}{ WBS Designator: 6.10 .14 .2} & Due Date: $\begin{array}{l}11 / 17 / 95 \\
05 / 17 / 96 \\
\end{array}$ \\
\hline \multicolumn{3}{|c|}{ Control Number: $C A-96-07$ A and B } & Revision: \\
\hline $\begin{array}{l}\text { Milestone Type: } \\
\square \text { DOE-HQ } \\
\square \text { DOE-RL } \\
\square \text { CNTR }\end{array}$ & $\begin{array}{ll} & \text { Division: } \\
\square & \text { State } \\
\square & \text { Federal } \\
\text { DOE } \\
\square \text { RCRA } \\
\square \text { TPA\# }\end{array}$ & $\begin{array}{l}\text { DELIVERABLE: } \\
\text { Report } \\
\square \text { Letter } \\
\square \text { Drawings } \\
\square \text { Other } \\
\text { (specify) }\end{array}$ & $\begin{array}{ll} & \text { ADDRESS TO: } \\
\square & \text { DOE-HQ } \\
\square & \text { DOE-RL } \\
\text { Other: ICF KH }\end{array}$ \\
\hline
\end{tabular}

\section{Milestone Description:}

ICF KH six-month evaluation of subcontract performance for fee determination.

Description of what constitutes completion of this milestone:

Completion occurs when the decisional is submitted to $\mathrm{RL}$ along with the request for written formal approval from $\mathrm{RL}$.

\begin{tabular}{|ll|ll|}
\hline $\begin{array}{l}\text { Cost Account Manager } \\
\text { J.M. Knoll, Jr. }\end{array}$ & Date & $\begin{array}{l}\text { Program/Project Manager } \\
\text { S. R. Morgan }\end{array}$ & Date \\
\hline $\begin{array}{l}\text { Program Element Manager } \\
\text { S. R. Morgan }\end{array}$ & Date & $\begin{array}{l}\text { DOE Monitor } \\
\text { A. W. Kellogg }\end{array}$ & Date \\
\hline
\end{tabular}




\begin{tabular}{|c|c|c|c|}
\hline Title: Prime Co & tract Transitio & & $\begin{array}{l}\text { Date Prepared: } \\
08 / 28 / 95 \\
\end{array}$ \\
\hline Assigned $\mathrm{To}^{\circ} \quad \mathrm{Co}_{\mathrm{O}}$ & tracts Administ & & CIN: \\
\hline WBS Designator: & 6.10 .14 .2 & & Due Date: $06 / 30 / 96$ \\
\hline Control Number: & CA-96-08 & & Revision: \\
\hline $\begin{array}{l}\text { Milestone Type: } \\
\square \text { DOE-HQ } \\
\square \text { DOE-RL } \\
\square \text { CNTR }\end{array}$ & $\begin{array}{ll} & \text { Division: } \\
\square & \text { State } \\
\square & \text { Federal } \\
& \text { DOE } \\
\square & \text { RCRA } \\
\square & \text { TPA\# }\end{array}$ & $\begin{array}{l}\text { DELIVERABLE: } \\
\square \text { Report } \\
\square \text { Letter } \\
\square \text { Drawings } \\
\text { Other: Plan }\end{array}$ & $\begin{array}{l}\text { ADDRESS TO: } \\
\square \text { DOE-HQ } \\
\text { DOE-RL } \\
\square \text { other } \\
\text { (specify) }\end{array}$ \\
\hline
\end{tabular}

\section{Milestone Description:}

Prime contract transition plan.

Description of what constitutes completion of this milestone:

Submittal of plan to RL.

\begin{tabular}{|lc|ll|}
\hline $\begin{array}{l}\text { Cost Account Manager } \\
\text { J. M. Knoll, Jr. }\end{array}$ & Date & $\begin{array}{l}\text { Program/Project Manager } \\
\text { S. R. Morgan }\end{array}$ & Date \\
\hline $\begin{array}{l}\text { Program Element Manager } \\
\text { S. R. Morgan }\end{array}$ & Date & $\begin{array}{l}\text { DOE Monitor } \\
\text { A. W. Kellogg }\end{array}$ & Date \\
\hline
\end{tabular}


Westinghouse Hanford Company

MILESTONE DESCRIPTION SHEET

Title: Prime Contract Settlement Proposal

\begin{tabular}{|l|l}
\hline Assigned To: Contracts Administration & CIN \\
\hline
\end{tabular}

WBS Designator: 6.10.14.2

Control Number: CA-96-09

Milestone Type:

$\square$ DOE-HQ

DOE-RL

CNTR

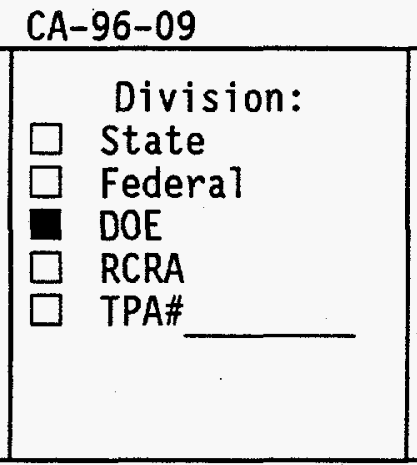

Due Date: 09/30/96

Milestone Description:

Prime contract settlement proposal.

Description of what constitutes completion of this milestone:

Submittal of proposal to RL.

\begin{tabular}{|ll|ll|}
\hline $\begin{array}{l}\text { Cost Account Manager } \\
\text { J.M. Knol1, Jr. }\end{array}$ & Date & $\begin{array}{l}\text { Program/Project Manager } \\
\text { S. R. Morgan }\end{array}$ & Date \\
\hline $\begin{array}{l}\text { Program Element Manager } \\
\text { S. R. Morgan }\end{array}$ & Date & $\begin{array}{l}\text { DOE Monitor } \\
\text { A. W. Kellogg }\end{array}$ & Date \\
\hline
\end{tabular}


Westinghouse Hanford Company

MILESTONE DESCRIPTION SHEET

\begin{tabular}{|c|c|c|c|}
\hline \multicolumn{3}{|c|}{$\begin{array}{l}\text { Title: Creation of at least one employee-owned or } \\
\text { locally-owned spinoff business performing work } \\
\text { at Hanford traditionally performed in-house }\end{array}$} & $\begin{array}{l}\text { Date Prepared: } \\
08 / 28 / 95\end{array}$ \\
\hline \multicolumn{3}{|c|}{ Assigned To: Contracts Administration } & CIN: \\
\hline \multicolumn{3}{|c|}{ WBS Designator: 6.10 .14 .2} & Due Date: $09 / 30 / 96$ \\
\hline \multicolumn{3}{|c|}{ Control Number: $\quad$ CA-96-10 } & Revision: \\
\hline $\begin{array}{l}\text { Milestone Type: } \\
\square \text { DOE-HQ } \\
\text { DOE-RL } \\
\square \text { CNTR }\end{array}$ & $\begin{array}{ll} & \text { Division: } \\
\square & \text { State } \\
\square & \text { Federal } \\
\text { DOE } \\
\square \text { RCRA } \\
\square \text { TPA\# }\end{array}$ & \begin{tabular}{|l} 
DELIVERABLE: \\
$\square$ Report \\
Letter \\
$\square$ Drawings \\
$\square$ Other \\
(specify)
\end{tabular} & $\begin{array}{l}\text { ADDRESS TO: } \\
\square \text { DOE-HQ } \\
\square \text { DOE-RL } \\
\square \text { Other } \\
\text { (specify) }\end{array}$ \\
\hline
\end{tabular}

\section{Milestone Description:}

Through the Make-or-Buy Review Board, develop strategies, enl ist employee and/or local investor support, and legally create spin-off subsidiaries of WHC with the express purpose of establishing economic diversification businesses to perform Hanford work traditionally performed by $M \& O$ in-house personnel. Primary preference will be to create such subsidiaries as a vehicle for establishing employee-owned businesses with a baseload contract for the work the employees have traditionally performed for the M\&O contractor. Where not feasible as an employee-owned business, WHC may maintain an equity position in such subsidiaries until local investors can be attracted to take over such businesses. All such businesses should become commercially viable within three years and be able to compete for private sector or Government work through other agencies.

Description of what constitutes completion of this milestone:

Incorporation of at least one WHC subsidiary and assignment of work through the award of subcontracts or IWR documents. Publication of a business plan outlining steps to be taken transferring equity to employee groups or local investors. Transfer of equity to such groups in FY 1996, while desired, shall not be a condition for milestone accomplishment.

\begin{tabular}{|ll|ll|}
\hline $\begin{array}{l}\text { Cost Account Manager } \\
\text { J. M. Knol1, Jr. }\end{array}$ & Date & $\begin{array}{l}\text { Program/Project Manager } \\
\text { S. R. Morgan }\end{array}$ & Date \\
\hline $\begin{array}{l}\text { Program Element Manager } \\
\text { S. R. Morgan }\end{array}$ & Date & $\begin{array}{l}\text { DOE Monitor } \\
\text { A. W. Kellogg }\end{array}$ & Date \\
\hline
\end{tabular}


MILESTONE DESCRIPTION SHEET

\begin{tabular}{|c|c|c|c|}
\hline \multicolumn{3}{|c|}{ Title: Scorecard Report } & Date: $10 / 25 / 95$ \\
\hline \multicolumn{3}{|c|}{ Assigned To: Cost Management Program Office } & CIN: \\
\hline \multicolumn{3}{|c|}{ Program WBS Designator: $6 \cdot 10 \cdot 14.3$} & $\begin{array}{l}\text { Due Date: } \\
11 / 30 / 95 \\
12 / 31 / 95 \\
\text { a11 others: TBD } \\
\end{array}$ \\
\hline \multicolumn{3}{|c|}{ Control Number: CMP-96-001A through L } & Rev: \\
\hline 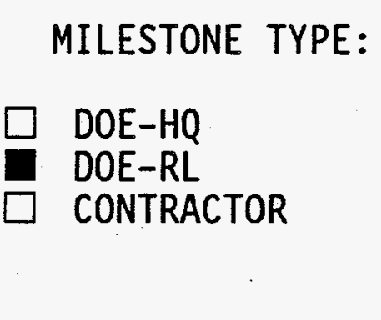 & \begin{aligned} & DIVISION: \\
& \multicolumn{1}{l}{} \\
&$\square$ State \\
&$\square$ Federal \\
& DOE \\
&$\square$ RCRA \\
&$\square$ TPA Number \end{aligned} & 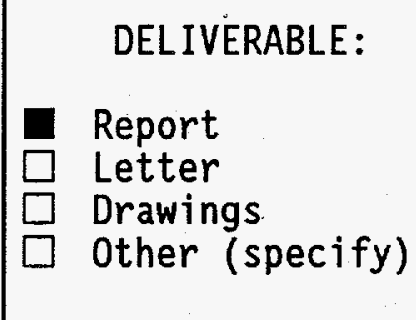 & $\begin{array}{l}\text { ADDRESS TO: } \\
\square \text { DOE-HQ } \\
\text { DOE-RL } \\
\square \text { Other } \\
\text { (specify) }\end{array}$ \\
\hline
\end{tabular}

\section{Milestone Description:}

A Savings Tracking System (Scorecard) Report will be provided to DOE-RL on a monthly basis. Submittal dates will be established when the FY 1996 format is approved.

Description of what constitutes completion of this milestone:

Delivery of the Scorecard Report to DOE-RL on a monthly basis. A schedule of submittal dates will be provided one week following RL approval of the FY 1996 format in December 1995.

\begin{tabular}{|lc|lc|}
\hline $\begin{array}{l}\text { Cost Account Manager } \\
\text { J. M. Knoll, Jr. }\end{array}$ & Date & $\begin{array}{l}\text { Program/Project Manager } \\
\text { J. R. Kirkendall }\end{array}$ & Date \\
\hline $\begin{array}{l}\text { Program Element Manager } \\
\text { J. R. Kirkendal1 }\end{array}$ & Date & $\begin{array}{l}\text { DOE Monitor } \\
\text { R. J. Light }\end{array}$ & Date \\
\hline
\end{tabular}


Westinghouse Hanford Company

MILESTONE DESCRIPTION SHEET

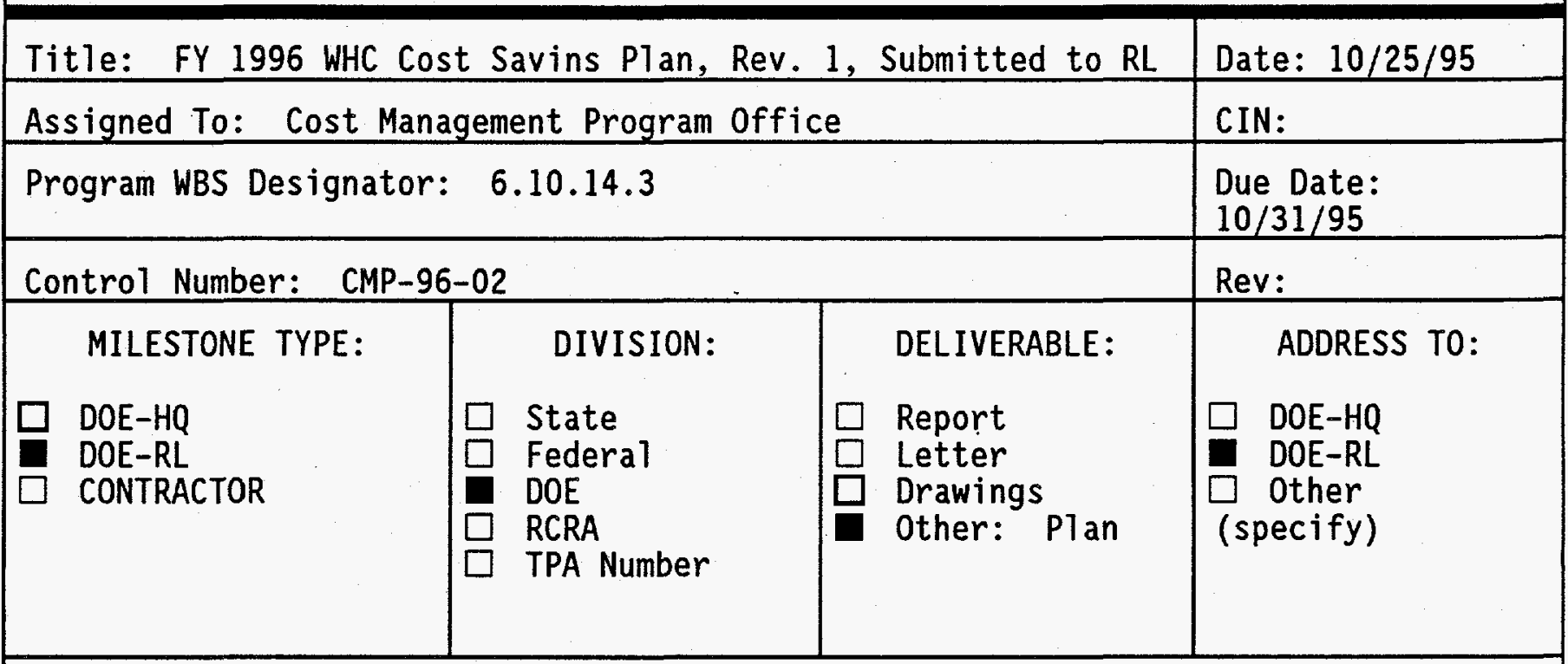

\section{Milestone Description:}

Prepare and submit the WHC Cost Savings Plan, Rev. 1, in accordance with RL comments and RL guidance letter, "Hanford Cost Savings Plan, " dated September 19, 1995.

Description of what constitutes completion of this milestone:

Delivery of $\mathrm{Pl}$ an to $\mathrm{RL}$.

\begin{tabular}{|lc|lc|}
\hline $\begin{array}{l}\text { Cost Account Manager } \\
\text { J.M. Knoll, Jr. }\end{array}$ & Date & $\begin{array}{l}\text { Program/Project Manager } \\
\text { J. R. Kirkendal1 }\end{array}$ & Date \\
\hline $\begin{array}{l}\text { Program Element Manager } \\
\text { J. R. Kirkenda17 }\end{array}$ & Date & $\begin{array}{l}\text { DOE Monitor } \\
\text { R. J. Light }\end{array}$ & Date \\
\hline
\end{tabular}




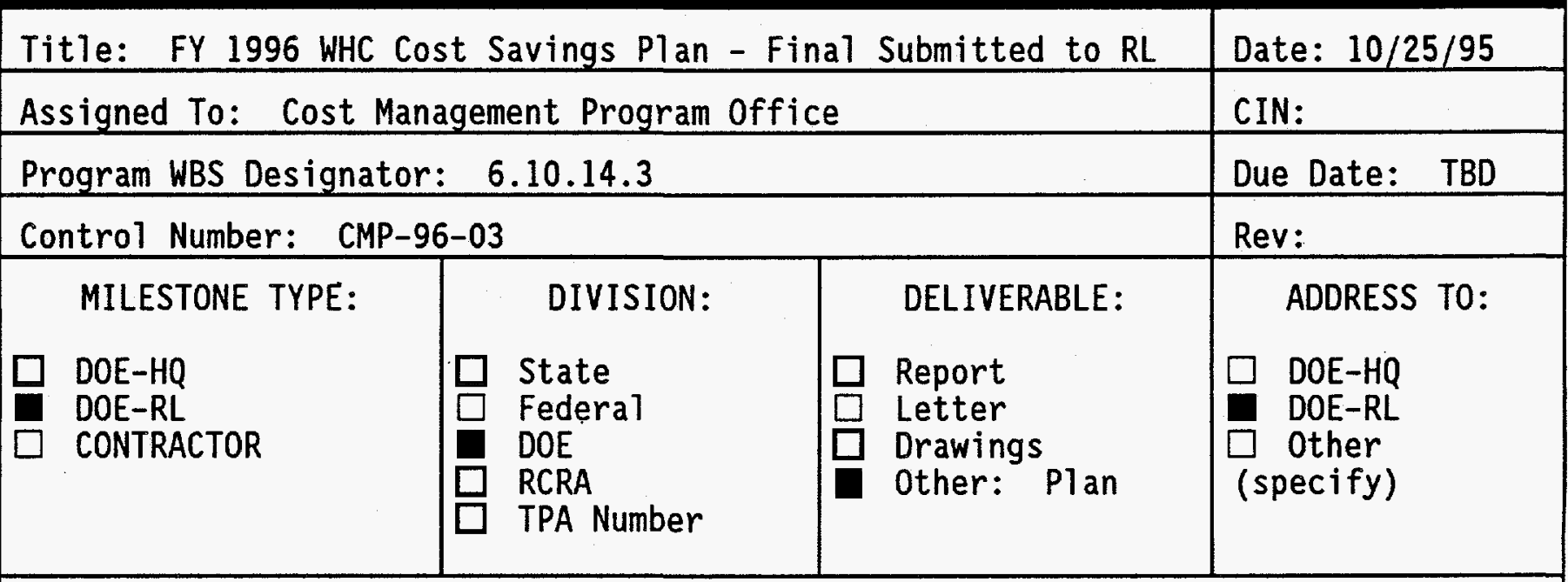

\section{Milestone Description:}

Prepare and submit the WHC Cost Savings Plan - Final, in accordance with RL comments and RL guidance letter, "Hanford Cost Savings Plan," dated September 19, 1995.

Description of what constitutes completion of this milestone:

Delivery of Plan to RL.

\begin{tabular}{|c|c|c|c|}
\hline $\begin{array}{l}\text { Cost Account Manager } \\
\text { J. M. Knol1, Jr. }\end{array}$ & Date & $\begin{array}{l}\text { Program/Project Manager } \\
\text { J. R. Kirkenda } 11\end{array}$ & Date \\
\hline $\begin{array}{l}\text { Program Element Manager } \\
\text { J. R. Kirkendall }\end{array}$ & Date & $\begin{array}{l}\text { DOE Monitor } \\
\text { R. J. Light }\end{array}$ & Date \\
\hline
\end{tabular}


Westinghouse Hanford Company MILESTONE DESCRIPTION SHEET

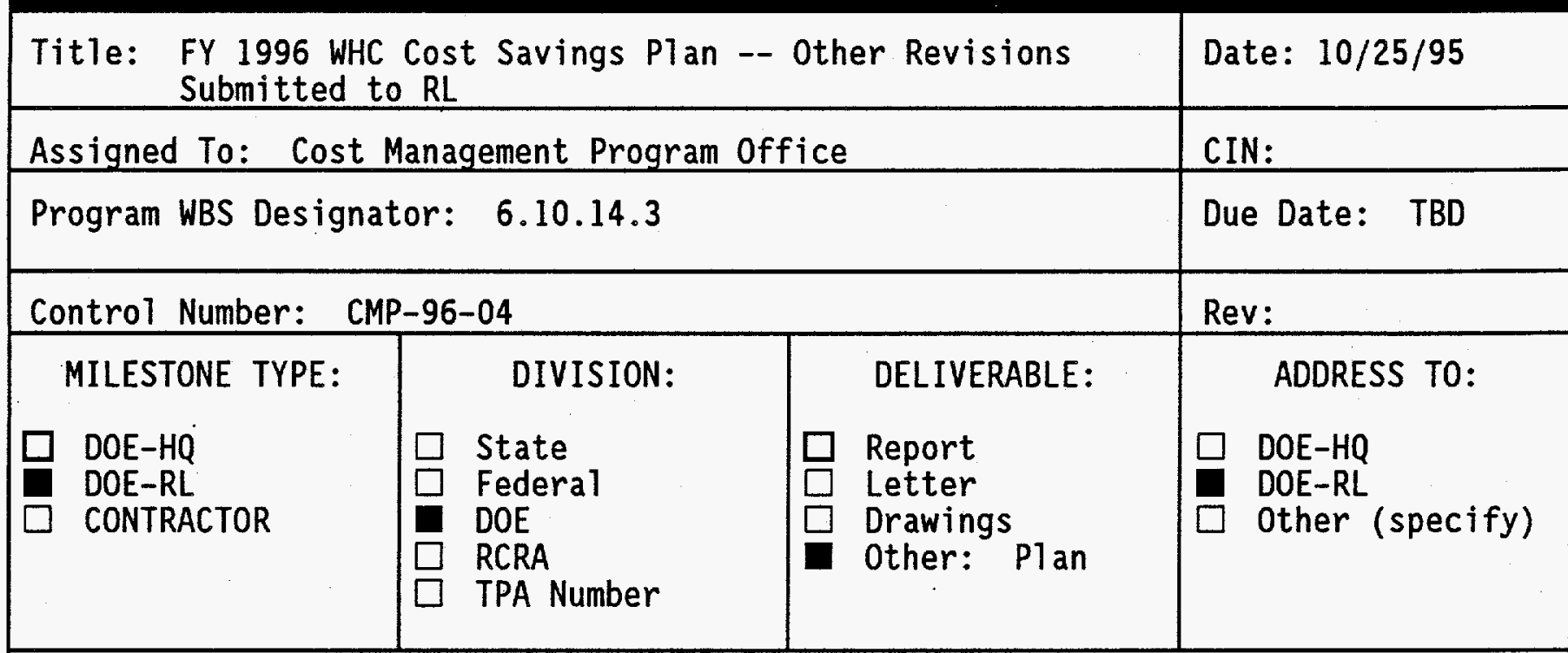

\section{Milestone Description:}

Prepare and submit the WHC Cost Savings Plan - Other Revisions, in accordance with RL comments.

Description of what constitutes completion of this milestone:

Delivery of $\mathrm{Pl}$ an to $\mathrm{RL}$.

Cost Account Manager

J. M. Knol1, Jr.

Program Element Manager

J. R. Kirkendall
Date Program/Project Manager J. R. Kirkendall

Date DOE Monitor

R. J. Light
Date

Date 
Westinghouse Hanford Company

MILESTONE DESCRIPTION SHEET

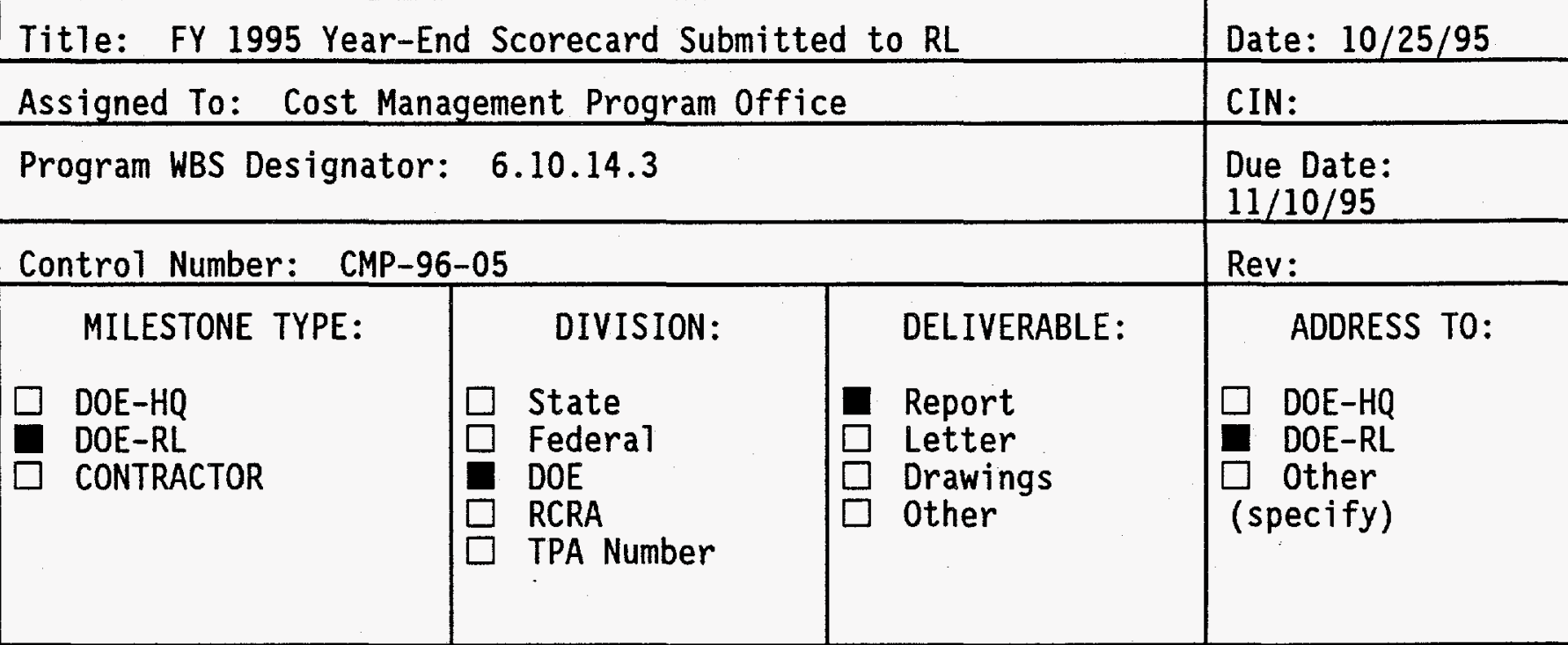

\section{Milestone Description:}

Submit the FY 1995 year-end Scorecard to RL to document total FY 1995 savings, including year-end efficiency savings as determined through the year-end reconciliation. Year-end efficiency savings may or may not match the adjusted cost variance used for fee payment. This Scorecard wi11 show the new FY 1996, FY 1997 and FY 1998 baselines from the approved September 1995 MYPPs.

Description of what constitutes completion of this milestone:

Delivery to RL.

\begin{tabular}{|ll|ll|}
\hline $\begin{array}{l}\text { Cost Account Manager } \\
\text { J.M. Knoll, Jr. }\end{array}$ & Date & $\begin{array}{l}\text { Program/Project Manager } \\
\text { J. R. Kirkendall }\end{array}$ & Date \\
\hline $\begin{array}{l}\text { Program Element Manager } \\
\text { J. R. Kirkendall }\end{array}$ & Date & $\begin{array}{l}\text { DOE Monitor } \\
\text { R. J. Light }\end{array}$ & Date \\
\hline
\end{tabular}


Westinghouse Hanford Company

MILESTONE DESCRIPTION SHEET

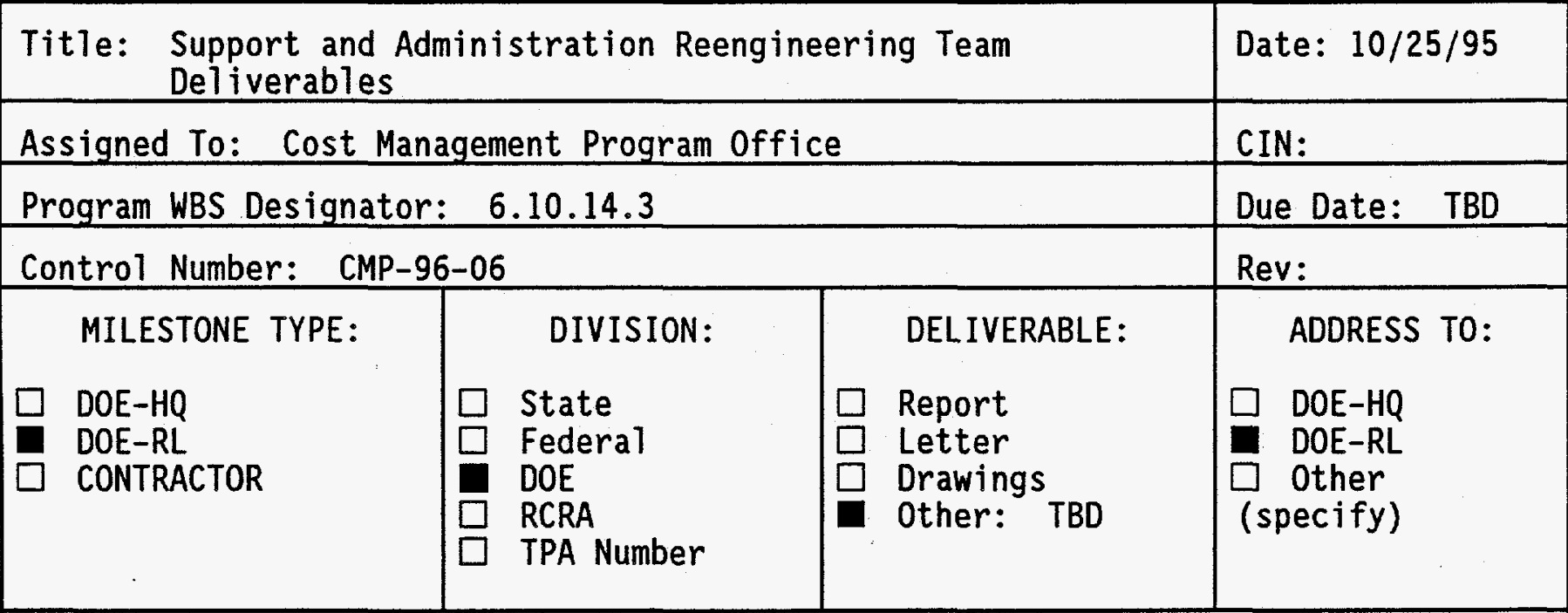

\section{Milestone Description:}

Milestones are being developed for each team and will be provided to RL by $10 / 31 / 95$.

Description of what constitutes completion of this milestone:

TBD

\begin{tabular}{ll|ll|}
$\begin{array}{l}\text { Cost Account Manager } \\
\text { J. M. Knoll, Jr. }\end{array}$ & Date & $\begin{array}{l}\text { Program/Project Manager } \\
\text { J. R. Kirkenda11 }\end{array}$ & Date \\
\hline $\begin{array}{l}\text { Program Element Manager } \\
\text { J. R. Kirkenda11 }\end{array}$ & Date & $\begin{array}{l}\text { DOE Monitor } \\
\text { R. J. Light }\end{array}$ & Date \\
\hline
\end{tabular}




\begin{tabular}{|c|c|c|c|c|}
\hline \multicolumn{5}{|c|}{$\begin{array}{l}\text { Westinghouse Hanford Company } \\
\text { MILESTONE DESCRIPTION SHEET }\end{array}$} \\
\hline \multicolumn{3}{|c|}{ Title: Plant Reengineering Team Deliverables } & \multicolumn{2}{|c|}{ Date: $10 / 25 / 95$} \\
\hline \multicolumn{3}{|c|}{ Assigned To: Cost Management Program Office } & \multicolumn{2}{|c|}{ CIN: } \\
\hline \multicolumn{3}{|c|}{ Program WBS Designator: $6 \cdot 10.14 .3$} & Due & TBD \\
\hline \multicolumn{3}{|c|}{ Control Number: CMP-96-07 } & \multicolumn{2}{|c|}{ Rev: } \\
\hline $\begin{aligned} & \text { MILESTONE TYPE: } \\
& \square \text { DOE-HQ } \\
& \text { DOE-RL } \\
& \square \text { CONTRACTOR }\end{aligned}$ & $\begin{aligned} & \text { DIVISION: } \\
& \square \text { State } \\
& \square \text { Federal } \\
& \text { DOE } \\
& \square \text { RCRA } \\
& \square \text { TPA Number }\end{aligned}$ & $\begin{aligned} & \text { DELIVERABLE: } \\
\square & \text { Report } \\
\square & \text { Letter } \\
\square & \text { Drawings } \\
& \text { Other: TBD }\end{aligned}$ & $\underset{\square}{(s)}$ & \\
\hline \multicolumn{5}{|c|}{$\begin{array}{l}\text { Milestone Description: } \\
\text { Milestones are being developed for each team and will be provided to RL by } \\
\text { 10/31/95. }\end{array}$} \\
\hline \multicolumn{5}{|c|}{$\begin{array}{l}\text { Description of what constitutes completion of this milestone: } \\
\text { TBD }\end{array}$} \\
\hline $\begin{array}{l}\text { Cost Account Manager } \\
\text { J. M. Knoll, Jr. }\end{array}$ & Date & $\begin{array}{l}\text { Program/Project } \\
\text { J. R. Kirkendall }\end{array}$ & & Date \\
\hline $\begin{array}{l}\text { Program Element Manag } \\
\text { J. R. Kirkenda11 }\end{array}$ & Date & $\begin{array}{l}\text { DOE Monitor } \\
\text { R. J. Light }\end{array}$ & & Date \\
\hline
\end{tabular}


Westinghouse Hanford Company

MILESTONE DESCRIPTION SHEET

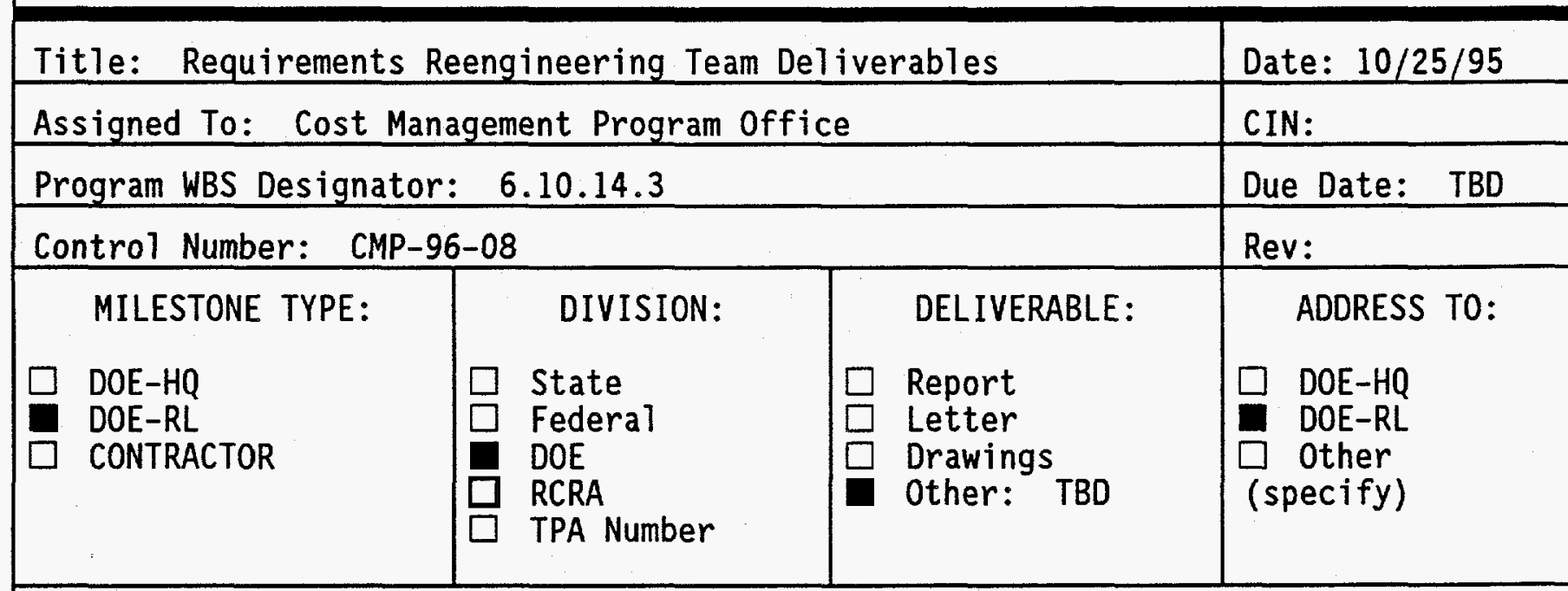

\section{Milestone Description:}

Milestones are being developed for each team and will be provided to RL by $10 / 31 / 95$.

Description of what constitutes completion of this milestone:

TBD

\begin{tabular}{|ll|lc|}
\hline $\begin{array}{l}\text { Cost Account Manager } \\
\text { J.M. Knoll, Jr. }\end{array}$ & Date & $\begin{array}{l}\text { Program/Project Manager } \\
\text { J. R. Kirkendall }\end{array}$ & Date \\
\hline $\begin{array}{l}\text { Program Element Manager } \\
\text { J. R. Kirkendall }\end{array}$ & Date & $\begin{array}{l}\text { DoE Monitor } \\
\text { R. J. Light }\end{array}$ & Date \\
\hline
\end{tabular}


FY 1996 Program P1 an

\section{Westinghouse Hanford Company}

MILESTONE DESCRIPTION SHEET

\begin{tabular}{|c|c|c|c|}
\hline \multicolumn{3}{|c|}{ Title: $\begin{array}{l}\text { Obtain RL Approval of the Final FY } 1996 \text { Scorecard } \\
\text { Format }\end{array}$} & Date: $10 / 25 / 95$ \\
\hline \multicolumn{3}{|c|}{ Assigned To: Cost Management Program Office } & CIN: \\
\hline \multicolumn{3}{|c|}{ Program WBS Designator: 6.10 .14 .3} & $\begin{array}{l}\text { Due Date: } \\
12 / 15 / 95\end{array}$ \\
\hline \multicolumn{3}{|c|}{ Control Number: CMP-96-09 } & Rev: \\
\hline $\begin{aligned} & \text { MILESTONE TYPE: } \\
& \square \text { DOE-HQ } \\
& \text { DOE-RL } \\
& \square \text { CONTRACTOR }\end{aligned}$ & $\begin{aligned} & \text { DIVISION: } \\
& \square \text { State } \\
& \square \text { Federal } \\
& \text { DOE } \\
& \square \text { RCRA } \\
& \square \text { TPA Number }\end{aligned}$ & \begin{tabular}{|l} 
DELIVERABLE: \\
$\square$ Report \\
$\square$ Letter \\
$\square$ Drawings \\
Other: cc:Mail
\end{tabular} & $\begin{aligned} & \text { ADDRESS TO: } \\
& \square \text { DOE-HQ } \\
& \text { DOE-RL } \\
& \text { Other (specify) }\end{aligned}$ \\
\hline
\end{tabular}

\section{Milestone Description:}

Finalize the FY 1996 Scorecard format and obtain RL approval.

Description of what constitutes completion of this milestone:

A cc:Mail from Kathy Andrews-Smith approving the FY 1996 Scorecard format will constitute completion of this milestone.

\begin{tabular}{|lc|lc|}
\hline $\begin{array}{l}\text { Cost Account Manager } \\
\text { J. M. Knol1, Jr. }\end{array}$ & Date & $\begin{array}{l}\text { Program/Project Manager } \\
\text { J. R. Kirkendal1 }\end{array}$ & Date \\
\hline $\begin{array}{l}\text { Program Element Manager } \\
\text { J. R. Kirkenda11 }\end{array}$ & Date & $\begin{array}{l}\text { DOE Monitor } \\
\text { R. J. Light }\end{array}$ & Date \\
\hline
\end{tabular}


Westinghouse Hanford Company

MILESTONE DESCRIPTION SHEET

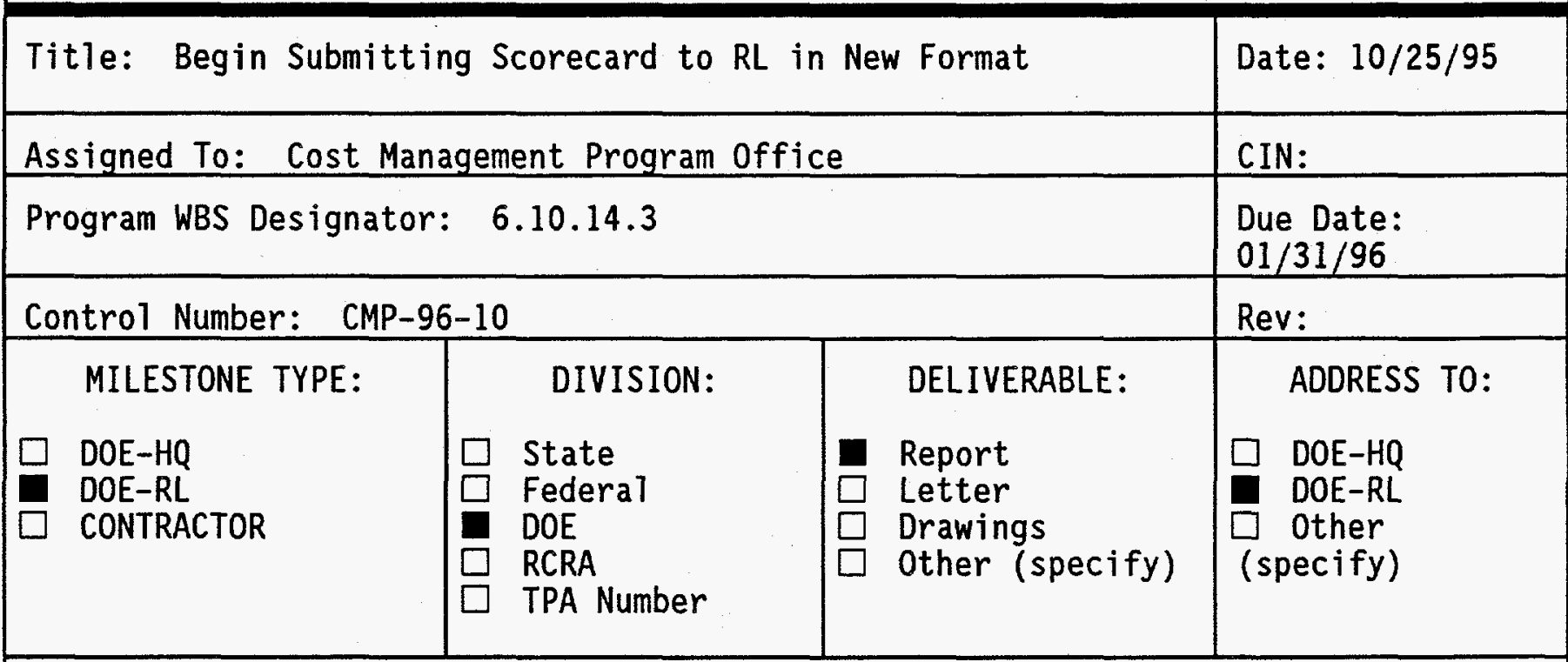

Milestone Description:

Submit the December 1995 Scorecard report to RL in the new format.

Description of what constitutes completion of this milestone:

Submittal of the report to RL.

Cost Account Manager

J. M. Knoll, Jr.

Program Element Manager

J. R. Kirkendal1
Date

Program/Project Manager J. R. Kirkendal]

Date DOE Monitor R. J. Light
Date

Date 


\begin{tabular}{|c|c|c|c|}
\hline \multicolumn{3}{|c|}{$\begin{array}{l}\text { Title: Implement Electronically-Generated Scorecard } \\
\text { (NOT FUNDED) }\end{array}$} & Date: $10 / 25 / 95$ \\
\hline \multicolumn{3}{|c|}{ Assigned To: Cost Management Program Office } & CIN: \\
\hline \multicolumn{3}{|c|}{ Program WBS Designator: 6.10 .14 .3} & Due Date: $12 / 15 / 95$ \\
\hline \multicolumn{3}{|c|}{ Control Number: CMP-96-11 } & Rev: \\
\hline $\begin{aligned} & \text { MILESTONE TYPE: } \\
\square & \text { DOE-HQ } \\
& \text { DOE-RL } \\
\square & \text { CONTRACTOR }\end{aligned}$ & $\begin{aligned} & \text { DIVISION: } \\
\square & \text { State } \\
\square & \text { Federal } \\
\square & \text { DOE } \\
\square & \text { RCRA } \\
\square & \text { TPA Number }\end{aligned}$ & $\begin{array}{ll} & \text { DELIVERABLE: } \\
& \text { Report } \\
\square & \text { Letter } \\
\square & \text { Drawings } \\
\square & \text { Other (specify) }\end{array}$ & $\begin{aligned} & \text { ADDRESS TO: } \\
\square & \text { DOE-HQ } \\
& \text { DOE-RL } \\
\square & \text { Other. (specify) }\end{aligned}$ \\
\hline
\end{tabular}

\section{Milestone Description:}

Complete development of the electronically-generated Scorecard, including electronic interface with other systems as appropriate.

Description of what constitutes completion of this milestone:

Demonstration of the electronic system constitutes completion of this milestone.

THIS MILESTONE IS NOT FUNDED IN THIS PROGRAM PLAN.

\begin{tabular}{|ll|ll|}
\hline $\begin{array}{l}\text { Cost Account Manager } \\
\text { J. M. Knoll, Jr. }\end{array}$ & Date & $\begin{array}{l}\text { Program/Project Manager } \\
\text { J. R. Kirkendall }\end{array}$ & Date \\
\hline $\begin{array}{l}\text { Program Element Manager } \\
\text { J. R. Kirkendall }\end{array}$ & Date & $\begin{array}{l}\text { DOE Monitor } \\
\text { R. J. Light }\end{array}$ & Date \\
\hline
\end{tabular}




\begin{tabular}{|c|c|c|c|}
\hline \multicolumn{3}{|c|}{$\begin{array}{l}\text { Title: Provide FY } 1995 \text { Year-End C-170 Fee Reconciliation } \\
\text { to RL }\end{array}$} & Date: $10 / 25 / 95$ \\
\hline \multicolumn{3}{|c|}{ Assigned To: Cost Management Program office } & CIN: \\
\hline \multicolumn{3}{|c|}{ Program WBS Designator: 6.10 .14 .3} & Due Date: $11 / 10 / 95$ \\
\hline \multicolumn{3}{|c|}{ Control Number: CMP-96-12 } & Rev: \\
\hline $\begin{aligned} & \text { MILESTONE TYPE: } \\
\square & \text { DOE-HQ } \\
& \text { DOE-RL } \\
\square & \text { CONTRACTOR }\end{aligned}$ & $\begin{aligned} & \text { DIVISION: } \\
& \square \text { State } \\
& \square \text { Federal } \\
& \text { DOE } \\
& \square \text { RCRA } \\
& \square \text { TPA Number }\end{aligned}$ & $\begin{array}{ll} & \text { DELIVERABLE: } \\
\square & \text { Report } \\
\square & \text { Letter } \\
\square & \text { Drawings } \\
\square & \text { Other (specify) }\end{array}$ & $\begin{aligned} & \text { ADDRESS TO: } \\
\square & \text { DOE-HQ } \\
& \text { DOE-RL } \\
\square & \text { Other (specify) }\end{aligned}$ \\
\hline
\end{tabular}

\section{Milestone Description:}

Provide the FY 1995 tear-end C-170 Fee Reconciliation by program, including Efficiency PARs (PARs without Change Requests) that equal the adjusted cost variance.

Description of what constitutes completion of this milestone:

Transmittal to RL of the reconcilication and Efficiency PARs by program.

\begin{tabular}{|lc|lc|}
\hline $\begin{array}{l}\text { Cost Account Manager } \\
\text { J.M. Knoll, Jr. }\end{array}$ & Date & $\begin{array}{l}\text { Program/Project Manager } \\
\text { J. R. Kirkendall }\end{array}$ & Date \\
\hline $\begin{array}{l}\text { Program Element Manager } \\
\text { J. R. Kirkendall }\end{array}$ & Date & $\begin{array}{l}\text { DOE Monitor } \\
\text { R. J. Light }\end{array}$ & Date \\
\hline
\end{tabular}




\begin{tabular}{|c|c|c|c|c|c|c|c|c|}
\hline \multicolumn{2}{|c|}{ Planned Staffing (Full Time Equivalent) } & \multicolumn{2}{|c|}{ FUNDED + UNFUNDED $=$ TOTAL } & \multicolumn{5}{|c|}{ Note: Job Family only After 1997} \\
\hline Job ramily. & Number & & & & & & & \\
\hline Job Category & & 1996 & 1997 & 1998 & 1999 & 2000 & 2001 & 2002 \\
\hline \multicolumn{9}{|l|}{ MANAGERS: } \\
\hline First line & M020 & $3.6+0.2=3.8$ & 3.8 & & & & & \\
\hline Generat/executive & MO20 & $1.0+0=1.0$ & 1.0 & & & & & \\
\hline \multicolumn{9}{|l|}{ Projec/Program } \\
\hline \multicolumn{9}{|l|}{ other } \\
\hline Subtotal & & $4.6+0.2=4.8$ & 4.8 & 4.8 & 4.8 & 4.8 & 4.8 & 4.8 \\
\hline \multicolumn{9}{|l|}{ ENGINEERS } \\
\hline \multicolumn{9}{|l|}{ Chemical } \\
\hline \multicolumn{9}{|l|}{ Civil } \\
\hline Computer & E030 & $5.0+1.0=6.0$ & 6.0 & & & & & \\
\hline \multicolumn{9}{|l|}{ Electrical } \\
\hline \multicolumn{9}{|l|}{ Environmental } \\
\hline Industrial & $E 060$ & $3.0+0.6=3.6$ & 3.6 & & & & & \\
\hline Mechanical & E070 & $1.0+0=1.0$ & 1.0 & & & & & \\
\hline \multicolumn{9}{|l|}{ Nuclear } \\
\hline \multicolumn{9}{|l|}{ Petroleum/Mining } \\
\hline \multicolumn{9}{|l|}{ Plant } \\
\hline \multicolumn{9}{|l|}{ Qual ity Control } \\
\hline \multicolumn{9}{|l|}{ Safety } \\
\hline other & & $23.0+0=23.0$ & 23.0 & & & & & \\
\hline Subtotal & & $32.0+1.6=33.6$ & 33.6 & 33.6 & 33.6 & 33.6 & 33.6 & 33.6 \\
\hline
\end{tabular}




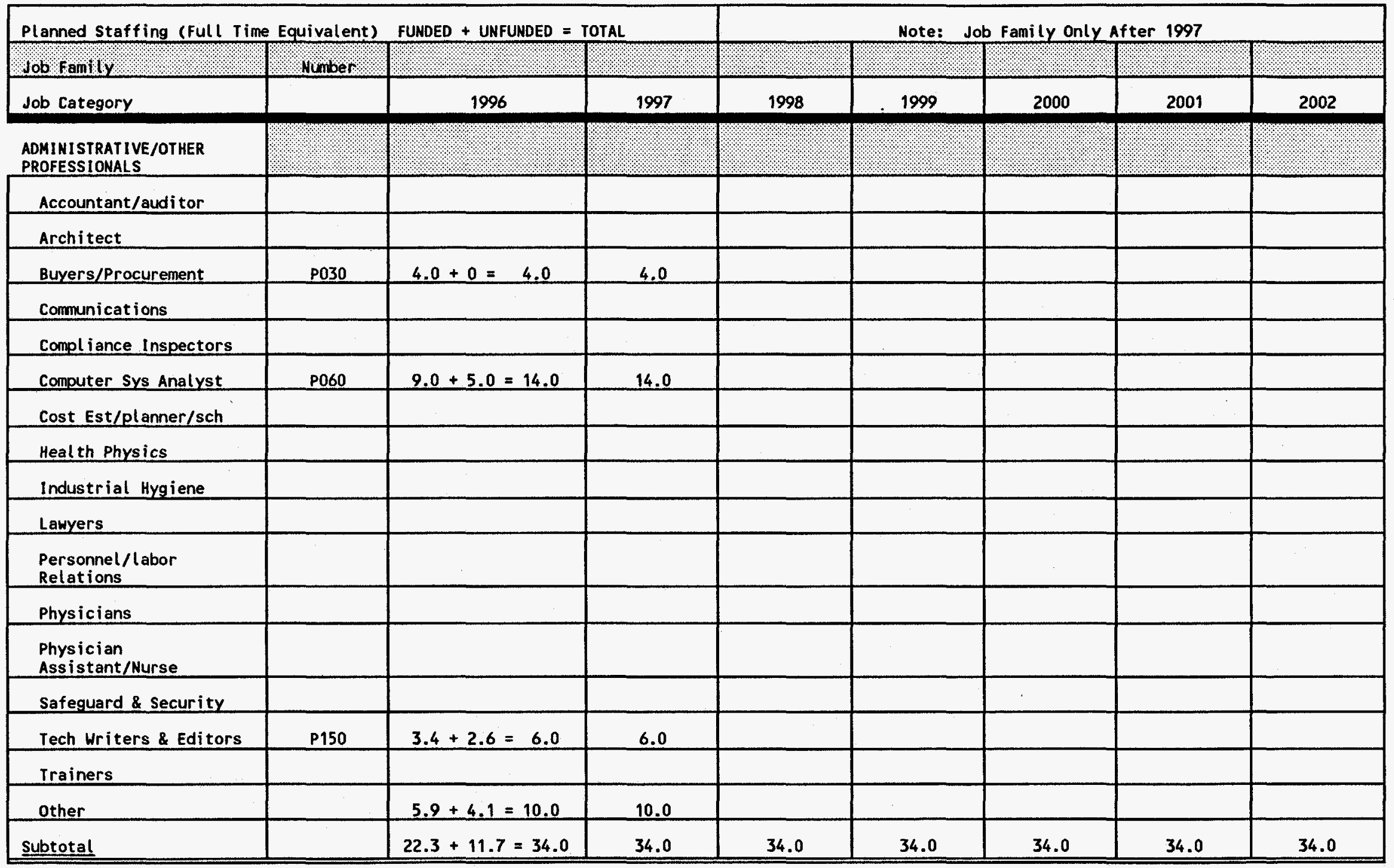




\begin{tabular}{|c|c|c|c|c|c|c|c|c|}
\hline Planned Staffing (Full Time & Equiva(ent) & FUNDED + UNFUNDED $=$ & TOTAL & & Note: & Job Family Only & After 1997 & \\
\hline ob r ramily & Number & & & & & & & \\
\hline Job Category & & 1996 & 1997 & 1998 & 1999 & 2000 & 2001 & 2002 \\
\hline \multicolumn{9}{|l|}{ GENERAL } \\
\hline Admin Assistant & G010 & $0.8+1.2=2.0$ & 2.0 & & & & & \\
\hline Office Clerk (Gen) & 6020 & $2.0+0=2.0$ & 2.0 & & & & & \\
\hline \multicolumn{9}{|l|}{ Office clerk (Special) } \\
\hline Secretaries & 6040 & $4.3+0=4.3$ & 4.3 & & & & & \\
\hline \multicolumn{9}{|l|}{ Iypist/Word Processor } \\
\hline \multicolumn{9}{|l|}{ other } \\
\hline Subtotal & & $7.1+1.2=8.3$ & 8.3 & 8.3 & 8.3 & 8.3 & 8.3 & 8.3 \\
\hline TOTAL FTES & & $66.0+14.7=80.7$ & 80.7 & 80.7 & 80.7 & 80.7 & 80.7 & 80.7 \\
\hline
\end{tabular}




\begin{tabular}{|c|c|c|c|c|c|c|c|c|c|}
\hline $\begin{array}{l}\text { Work } \\
\text { Breakdown } \\
\text { Structure } \\
\text { Dictionary }\end{array}$ & \multicolumn{6}{|c|}{$\begin{array}{c}\text { Westinghouse Hanford Company } \\
\text { (insert responsible organization title here) } \\
\text { Part I - Summary } \\
\text { (Dollars in 000's) }\end{array}$} & \multicolumn{3}{|c|}{$\begin{array}{l}\text { FY } 1996 \\
\text { Indirect } \\
\text { Program Plan } \\
\text { Rev. \# } 0 \\
\text { 25-Aug-95 }\end{array}$} \\
\hline $\begin{array}{c}\text { Cost Account Number } \\
\text { 1MDD42 } \\
\end{array}$ & \multicolumn{6}{|c|}{$\begin{array}{l}\text { Cost Account Title } \\
\text { CONTRACTS \& MANAGEMENT SERVICES }\end{array}$} & \multicolumn{3}{|c|}{$\begin{array}{l}\text { Proposed Rate: } \\
\text { (Rated Service Pool Only) }\end{array}$} \\
\hline $\begin{array}{r}\text { SMS WBS Number } \\
6.10 .14 \\
\end{array}$ & \multicolumn{6}{|c|}{$\begin{array}{l}\text { SMS Title } \\
\text { CONTRACTS \& MANAGEMENT SERVICES }\end{array}$} & \multicolumn{3}{|c|}{$\begin{array}{l}\text { Funding Source: } \\
x\end{array}$} \\
\hline \multicolumn{7}{|l|}{ RL SMS Program Manger } & \multicolumn{3}{|l|}{ Sws } \\
\hline Cost Account Manager & \multicolumn{6}{|l|}{ JM Knoll } & \multicolumn{3}{|l|}{ OST } \\
\hline \multicolumn{7}{|l|}{ WHC SMS Program Manager } & \multicolumn{3}{|l|}{$\mathrm{DOH}$} \\
\hline Financial Manager & \multicolumn{6}{|l|}{ DL Borders } & \multicolumn{3}{|l|}{ MGT PRO } \\
\hline \multirow[t]{2}{*}{ Responsible Analyst } & \multicolumn{6}{|l|}{ Judy Stewart } & \multirow{2}{*}{\multicolumn{3}{|c|}{\begin{tabular}{|l|} 
POOL \\
\\
FY 1996 Baseline \\
Full-Time Equivalents (FTEs)
\end{tabular}}} \\
\hline & \multicolumn{3}{|c|}{$\begin{array}{l}\text { FY } 1996 \text { Approved Funding } \\
\text { Full-Time Equivalents (FTEs) }\end{array}$} & \multicolumn{3}{|c|}{$\begin{array}{l}\text { FY } 1996 \text { Unfunded } \\
\text { Full-Time Equivalents (FTEs) }\end{array}$} & & & \\
\hline TYPE OF FTE & Exempt & Non-Exempt & Bargaining & Exempt & Non-Exempt & Bargaining & Exempt & Non-Exempt & Bargaining \\
\hline Organizational & 28.2 & 3.3 & & 13.7 & & & 41.9 & 3.3 & 0 \\
\hline Support & 3 & 0 & & 1 & & & 4 & 0 & 0 \\
\hline TOTALFTES & 31.2 & 3.3 & 0 & 14.7 & 0 & 0 & 45.9 & 3.3 & 0 \\
\hline COST ELEMENTS & \multicolumn{3}{|c|}{ FY 1996 Approved Funding Budget } & \multicolumn{3}{|c|}{ FY 1996 Unfunded Budget } & \multicolumn{3}{|c|}{ FY 1996 Baseline Budget } \\
\hline Labor - Regular & & & 2281.4 & & & 941 & & & 3222 \\
\hline Labor - Overtime & & & 11 & & & & & & 1 \\
\hline 0 Total Labor & & & 2282.4 & & & 940.6 & & & 3223 \\
\hline 1 Materials & & & 55 & & & 22.1 & & & 77.1 \\
\hline 2 Purchased Services & & & 244.8 & & & 82.7 & & & 327.5 \\
\hline 3 Other Hanford & & & 3.7 & & & 0.5 & & & 4.2 \\
\hline Subtotal Originated Costs & & & 2585.9 & & & 1045.9 & & & 3631.8 \\
\hline 4 Site Services & & & 284.6 & & & & & & 284.6 \\
\hline 5 Internal Charges & & & 16 & & & & & & 16 \\
\hline 6 IRM Support & & & 440.5 & & & & & & 440.5 \\
\hline 7 Overheads & & & 87.8 & & & 43.9 & & & 131.7 \\
\hline 8 Revenue & & & & & & - & & & 0 \\
\hline TOTAL DOLLARS & 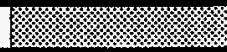 & & 3414.8 & : & & 1089.8 & & & 4504.6 \\
\hline
\end{tabular}

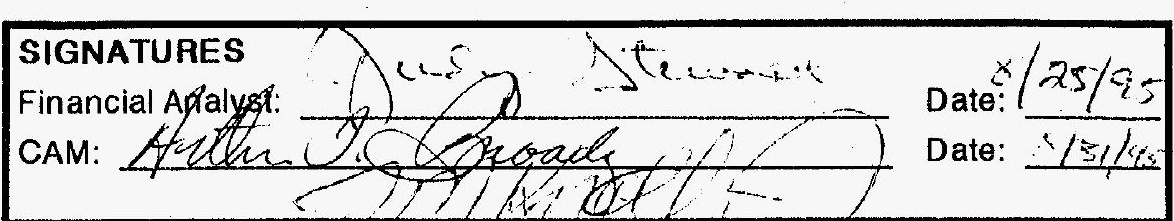

BASELINE APPROVAL




\begin{tabular}{|c|c|c|c|c|c|c|c|c|c|}
\hline $\begin{array}{l}\text { Work } \\
\text { Breakdown } \\
\text { Structure } \\
\text { Dictionary }\end{array}$ & \multicolumn{6}{|c|}{$\begin{array}{c}\text { Westinghouse Hanford Company } \\
\text { (insert responsible organization title here) } \\
\text { Part I - Summary } \\
\left.\text { (Dollars in } 000^{\prime} s\right)\end{array}$} & \multicolumn{3}{|c|}{$\begin{array}{l}\text { FY } 1996 \\
\text { Indirect } \\
\text { Program Plan } \\
\text { Rev. \# } 0 \\
\text { 25-Aug-95 }\end{array}$} \\
\hline $\begin{array}{l}\text { Cost Account Num ber } \\
\text { 1MDD } 42 \text { * }\end{array}$ & \multicolumn{6}{|c|}{$\begin{array}{l}\text { Cost ACCOUnt TIII } \\
\text { COST REDUCTION PROGRAM (*WORK PACKAGE WITHIN CONTRACTS MANAGEMENT) }\end{array}$} & \multicolumn{3}{|c|}{$\begin{array}{l}\text { Proposed Rate: } \\
\text { (Batod Sorvice Pool Only) }\end{array}$} \\
\hline $\begin{array}{r}\text { SMS WBS Number } \\
6.10 .14 \mathrm{X}\end{array}$ & \multicolumn{6}{|c|}{$\begin{array}{l}\text { SMS TIIIO } \\
\text { COST REDUCTON PROGRAM }\end{array}$} & \multicolumn{3}{|c|}{ Funding Sourco: } \\
\hline RL SMS Program Manger & \multicolumn{6}{|c|}{ AJ Light } & \multicolumn{3}{|l|}{ sws } \\
\hline Cost Account Manager & \multicolumn{6}{|l|}{ JM Knoll } & \multicolumn{3}{|l|}{ OST } \\
\hline WHC SMS Program Manager & \multicolumn{6}{|l|}{ er JM Knoll } & \multicolumn{3}{|l|}{$\mathrm{DOH}$} \\
\hline Financlal Manager & \multicolumn{6}{|l|}{ DLBorders } & \multicolumn{3}{|l|}{ MGT PRO } \\
\hline Responsible Analyst & \multicolumn{6}{|l|}{ Judy Stowant } & \multicolumn{3}{|l|}{ POOL } \\
\hline 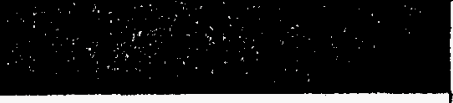 & \multicolumn{3}{|c|}{$\begin{array}{l}\text { FY } 1996 \text { Approved Funding } \\
\text { Full-Timo Equivalonts (FTEs) }\end{array}$} & \multicolumn{3}{|c|}{$\begin{array}{l}\text { FY } 1996 \text { Unfunded } \\
\text { Full-Time Equivalents (FTEs) }\end{array}$} & \multicolumn{3}{|c|}{$\begin{array}{c}\text { FY } 1996 \text { B aseline } \\
\text { Full-Time Equivalonts (FTEs) }\end{array}$} \\
\hline TYPE OF FTE & Exempt & Non-Exompt & Bargaining & Exompt & Non-Exempt & Bargaining & Exempt & Non-Exempt & Bargaining \\
\hline Organizational & 6.2 & 0.8 & 0 & 4.6 & 0 & 0 & 10.8 & 0.8 & $\underline{0}$ \\
\hline Support & 0 & 0 & 0 & 0 & 0 & 0 & 0 & 0 & 0 \\
\hline TOTAL FTES & 6.2 & 0.8 & 0 & 4.6 & 0 & 0 & 10.8 & 0.8 & 0 \\
\hline COST ELEMENTS & \multicolumn{3}{|c|}{ FY 1996 Approved Funding Budgot } & FY 19 & 96 Unfunded $B$ & udgot & \multicolumn{3}{|c|}{ FY 1996 B aseline Budget } \\
\hline Labor - Regular & & & 456 & & & 317 & & & 772.9 \\
\hline Labor - Overtime & & & & & & & & & 0 \\
\hline 0 Total Labor & & & 456 & & & 316.9 & & & 772.9 \\
\hline 1 Materials & & & 8 & & & 3.7 & & $\sqrt[3]{1+12}$ & 11.7 \\
\hline 2 Purchased Services & & & 86 & & ?. & 41.1 & & & 127.1 \\
\hline 3 Other Hanford & & & 1 & & & 0.5 & & 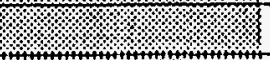 & 1.5 \\
\hline Sublotal Origin atod Costs & & & \%ै 551 & & & $3 \longdiv { 3 6 2 . 2 }$ & & & $\mathbf{9 1 3 . 2}$ \\
\hline 4 Sile Services & & & 14.5 & & & & & & 14.5 \\
\hline 5 Internal Charges & & & & & & & & & 0 \\
\hline 6 IRM Support & & & 39.3 & & 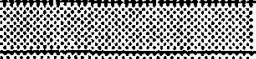 & & & & 39.3 \\
\hline 7 Overheads & & & & & & & & $\sqrt{1+1}$ & 0 \\
\hline 8 Revenue & 19.1.:. & 12.1. & & / & 1. & & $12.1 \%$ & $1 \%$ & 0 \\
\hline TOTAL DOLLARS & (3) & 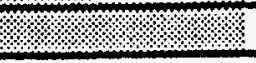 & 604.8 & 1/:- & m. & 362.2 & l' & স & 967 \\
\hline $\begin{array}{l}\text { SIGNATURES } \\
\text { Financial Analyst: } \\
\text { CAM: }\end{array}$ & & & $\begin{array}{l}\text { Date: } \\
\text { Date: }\end{array}$ & & $\longdiv { \text { BASELINE AP } }$ & PROVAL & & & Date: \\
\hline
\end{tabular}




\section{Cost Account Narrative Summary}

Provide company level direction in the areas of: contracts administration; cost reduction and cost-effectiveness programs; liability management; Directives and waivers; performance evaluation; controlled manual system; data management practices; Hanford Action Tracking System (HATS).

\section{Approved Funding Budget:}

Activity Detailed Description

Director and Executive Secretary, Contracts and Management Services

2.0 FTEs; $\$ 107$ originated cost plus assessments such as occupancy

\begin{tabular}{l}
$\qquad$ Total Activity Cost \\
\hline Activity Detailed Description \\
Contracts Administration \\
- Contract Reform, Performance-Based Initiatives, Make-or-Buy \\
- Subcontract Enforcement \\
- Subcontractor Performance Evaluation and Motivation \\
Prime Contract Proposals, Negotiations, and Claims \\
Project Management \\
Prime Contract Cost Administration and Liability Claim Defense \\
Clerical Support
\end{tabular}

10.0 FTEs; $\$ 748$ originated cost plus assessments such as occupancy 
Part II Activity Detail

Cost Account No. IMDD42

Activity Detailed Description

Cost Management Program

- Administer cost reduction incentive program

- Produce RL-directed Savings Scorecard

- Cost Management Program Board Meetings

7.0 FTEs; $\$ 551$ originated cost plus assessments such as occupancy

\section{Total Activity Cost}

Activity Detailed Description

Contract Requirements Integration

- $\quad$ Site Management System (SMS) Reporting

- $\quad$ Site Support Program Plan (SSPP)

- Liability Management

- Directives and Waivers

- Performance Evaluation

- Establish and manage Controlled Manual System

7.5 FTEs; $\$ 553.9$ originated cost plus assessments such as occupancy

$$
\text { Total Activity Cost }
$$

Activity Detailed Description

Implement Improved Data Management Practices

2.0 FTEs; $\$ 142$ originated cost plus assessments such as occupancy

Total Activity Cost 
Activity Detailed Description

Manage Hanford Action Tracking System (HATS)

4.0 FTES (3.0 HATS, 1.0 SHARE), 2.0 support FTEs;

$\$ 484$ originated cost plus assessments such as occupancy

Total Approved Funding Originated Budget

Total Approved Funding Non-Originated Budget

828.9

Total Approved Funding Budget 
Part II Activity Detail

Cost Account No. 1MDD42

Unfunded Budget:

Activity Detailed Description

Contracts Administration -- Subscriptions, professional appointments, and memberships.

0.25 FTES

$$
\text { Total Activity Cost }
$$

Activity Detailed Description

Cost Management Program

- Complete electronically-generated Scorecard

4.6 FTES

Total Activity Cost

Activity Detailed Description

Contract Requirements Integration

- Headquarters Building Management

- Award Fee Point of Contact

- Controlled Manual Point of Contact

- Certification Support

- Direct Company Controlled Manual System

4.85 FTES

Total Activity Cost

FY 1996

\begin{tabular}{|l|l|}
\hline \multicolumn{2}{|c|}{ FY 1996} \\
\hline FTEs & Dollars \\
\hline & \\
& \\
\hline 0.25 & \\
\hline
\end{tabular}


Part II Activity Detail

Cost Account No. 1MDD42

Activity Detailed Description

RL-Directed SHARING CONFERENCE

0 FTES

Total Activity Cost

\section{Activity Detailed Description}

Contracts Data Management and Integration

- Integrate sitewide strategic information planning

- Manage Data Administration Council

- Direct/Support TWRS Data Management Activities

- Manage Smart Cards

3.0 FTES

Total Activity Cost

\section{Activity Detailed Description}

Hanford Action Tracking System (HATS) -- Convert QUEST data

1.0 FTE, 1.0 support FTE

Total Activity Cost

2.0

Total Unfunded Originated Budget

Total Unfunded Non-Originated Budget

Total Unfunded Budget

Total Approved Funding \& Unfunded Budgets

\begin{tabular}{r|r|}
\hline 14.7 & $1,045.9$ \\
\hline & 43.9 \\
\hline 49.2 & $1,089.8$ \\
\hline
\end{tabular}




\begin{tabular}{|c|c|c|c|c|c|c|c|c|c|}
\hline $\begin{array}{l}\text { Work } \\
\text { Breakdown } \\
\text { Structure } \\
\text { Dictionary }\end{array}$ & \multicolumn{6}{|c|}{$\begin{array}{c}\text { Westinghouse Hanford Company } \\
\text { (insert responsible organization title here) } \\
\text { Part I - Summary } \\
\text { (Dollars in 000's) }\end{array}$} & \multicolumn{3}{|c|}{$\begin{array}{l}\text { FY } 1996 \\
\text { Indirect } \\
\text { Program Plan } \\
\text { Rev. \# } 0 \\
25-\text { Aug-95 }\end{array}$} \\
\hline $\begin{array}{c}\text { Cost Account Number } \\
\text { 1MDBPC }\end{array}$ & \multicolumn{6}{|c|}{$\begin{array}{l}\text { Cost Account Title } \\
\text { PEOPLECORE / DS / HISI }\end{array}$} & \multicolumn{3}{|c|}{$\begin{array}{l}\text { Proposed Rate: } \\
\text { (Rated Service Pool Only) }\end{array}$} \\
\hline $\begin{array}{r}\text { SMS WBS Number } \\
6.10 .14 \\
\end{array}$ & \multicolumn{6}{|c|}{$\begin{array}{l}\text { SMS Title } \\
\text { CONTRACTS \& MANAGEMENT SERVICES }\end{array}$} & \multicolumn{3}{|c|}{ Funding Source: } \\
\hline \multicolumn{7}{|l|}{ RL SMS Program Manger } & \multicolumn{3}{|l|}{ sws } \\
\hline Cost Account Manager & \multicolumn{6}{|l|}{ MK Britton } & \multicolumn{3}{|l|}{ OST } \\
\hline \multicolumn{7}{|l|}{ WHC SMS Program Manager } & \multicolumn{3}{|l|}{ DOH } \\
\hline Financial Manager & \multicolumn{6}{|l|}{ DL Borders } & \\
\hline \multirow[t]{2}{*}{ Responsible Analyst } & \multicolumn{6}{|l|}{ Judy Stewart } & \multicolumn{3}{|l|}{ POOL } \\
\hline & \multicolumn{3}{|c|}{$\begin{array}{l}\text { FY } 1996 \text { Approved Funding } \\
\text { Full-Time Equivalents (FTEs) }\end{array}$} & \multicolumn{3}{|c|}{$\begin{array}{l}\text { FY } 1996 \text { Unfunded } \\
\text { Full-Time Equivalents (FTEs) }\end{array}$} & \multicolumn{3}{|c|}{$\begin{array}{c}\text { FY } 1996 \text { Baseline } \\
\text { Full-Time Equivalents (FTEs) }\end{array}$} \\
\hline TYPE OF FTE & Exempt & Non-Exempt & Bargaining & Exempt & Non-Exempt & Bargaining & Exempt & Non-Exempt & Bargaining \\
\hline Organizational & 4.5 & 0.5 & & & & & 4.5 & 0.5 & 0 \\
\hline Support & 1 & 0 & & & & & 1 & 0 & $\mathbf{0}$ \\
\hline TOTALFTES & 5.5 & 0.5 & 0 & 0 & 0 & 0 & 5.5 & 0.5 & 0 \\
\hline COST ELEMENTS. & \multicolumn{3}{|c|}{ FY 1996 Approved Funding Budget } & \multicolumn{3}{|c|}{ FY 1996 Unfunded Budget } & \multicolumn{3}{|c|}{ FY 1996 Baseline Budget } \\
\hline . Labor - Regular & & & 501 & & & & & & 501 \\
\hline . Labor - Overtime & & & & & & & & & 0 \\
\hline 0 Total Labor & & & 501 & & & 0 & & & 501 \\
\hline 1 Materials & & & & & & & & & 0 \\
\hline 2 Purchased Services & & & & & & & & & 0 \\
\hline 3 Other Hanford & & & & & & & & & 0 \\
\hline Subtotal Originated Costs & & & 501 & & & 0 & & & 501 \\
\hline 4 Site Services & & & & & & & & & 0 \\
\hline 5 Internal Charges & & & & & & & & & 0 \\
\hline 6 IRM Support & & & 164.4 & & & & & & 164.4 \\
\hline 7 Overheads & & & 43.9 & & & & & & 43.9 \\
\hline 8 Revenue & & & & & & & & & 0 \\
\hline TOTAL DOLLARS & & & 709.3 & & & 0 & & & 709.3 \\
\hline
\end{tabular}

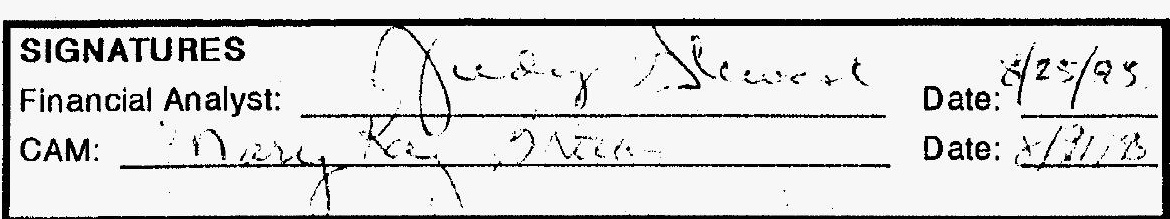

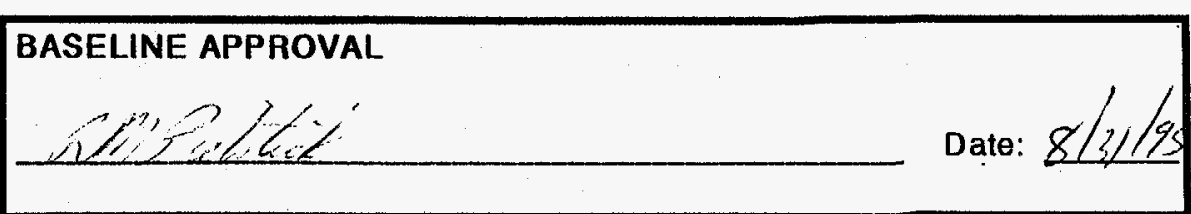




\section{Cost Account Narrative Summary}

Provide sitewide direction in the areas of Hanford PeopleCORE, Hanford Information System Inventory (HISI), and the site data standards process.

\section{Approved Funding Budget:}

Activity Detailed Description

Manage Hanford PeopleCORE

2.5 FTEs, 1.0 Support FTE

$$
\text { Total Activity Cost }
$$

Activity Detailed Description

Maintain Hanford Information System Inventory (HISI)

1.0 FTE

$$
\text { Total Activity Cost }
$$

Activity Detailed Description

Manage Site Data Standards Process

$$
1.5 \text { FTES }
$$

$$
\text { Total Activity Cost }
$$

1.5

$\$$

Total Approved Funding Originated Budget

Total Approved Funding Non-Originated Budget

Total Approved Funding Budget
6.0

$0 \quad 501.0$

FY 1996 Dollars 


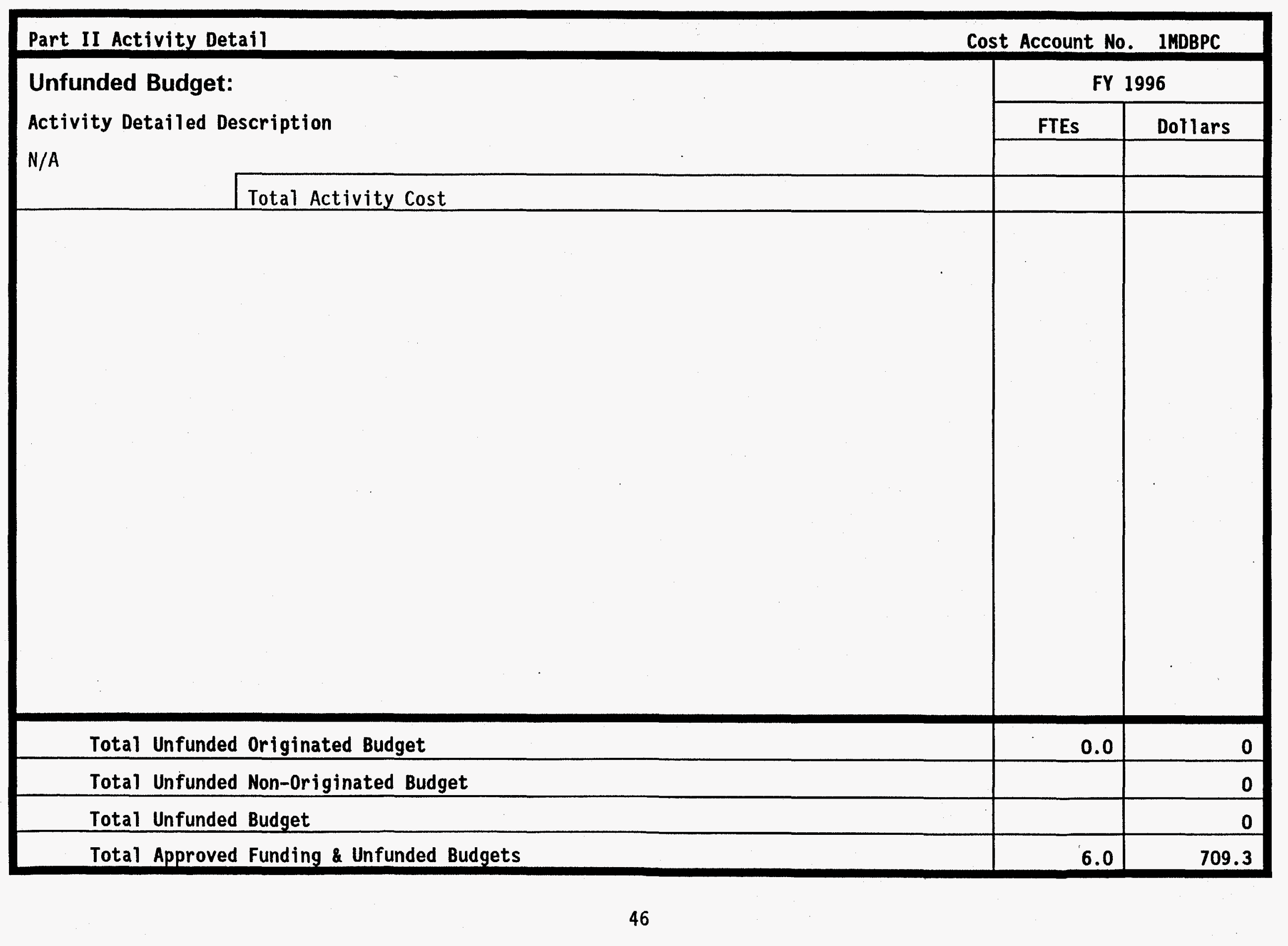




\begin{tabular}{|c|c|c|c|c|c|c|c|c|c|}
\hline $\begin{array}{l}\text { Work } \\
\text { Breakdown } \\
\text { Structure } \\
\text { Dictionary }\end{array}$ & \multicolumn{6}{|c|}{$\begin{array}{c}\text { Westinghouse Hanford Company } \\
\text { (insert responsible organization title here) } \\
\text { Part I - Sum mary } \\
\text { (Dollars in 000's) }\end{array}$} & \multicolumn{3}{|c|}{$\begin{array}{l}\text { FY } 1996 \\
\text { Indirect } \\
\text { Program Plan } \\
\text { Rev. \# } 0 \\
\text { 30-Aug-95 }\end{array}$} \\
\hline $\begin{array}{c}\text { Cost Account Number } \\
\text { 1MDDCs }\end{array}$ & \multicolumn{6}{|c|}{$\begin{array}{l}\text { Cost Account Title } \\
\text { REENGINEERING }\end{array}$} & \multicolumn{3}{|c|}{$\begin{array}{l}\text { Proposed Rate: } \\
\text { (Rated Service Pool Only) }\end{array}$} \\
\hline $\begin{array}{r}\text { SMS WBS Number } \\
6.10 .13 \\
\end{array}$ & \multicolumn{6}{|c|}{$\begin{array}{l}\text { SMS TIIle } \\
\text { REENGINEERING }\end{array}$} & \multicolumn{3}{|c|}{$\begin{array}{l}\text { Funding Source: } \\
X\end{array}$} \\
\hline \multicolumn{7}{|l|}{ RL SMS Program Manger } & \multicolumn{3}{|l|}{ SWS } \\
\hline Cost Account Manager & \multicolumn{6}{|c|}{ JR KIRKENDALL } & \multicolumn{3}{|l|}{ OST } \\
\hline \multicolumn{7}{|l|}{ WHC SMS Program Manager } & \multicolumn{3}{|l|}{$\mathrm{DOH}$} \\
\hline Financial Manager & \multicolumn{6}{|l|}{ DL Borders } & \multicolumn{3}{|l|}{ MGT PRO } \\
\hline \multirow[t]{2}{*}{ Responsible Analyst } & \multicolumn{6}{|l|}{ Judy Stowart } & \multicolumn{3}{|l|}{ POOL } \\
\hline & \multicolumn{3}{|c|}{$\begin{array}{l}\text { FY } 1996 \text { Approved Funding } \\
\text { Full-Time Equivalents (FTEs) }\end{array}$} & \multicolumn{3}{|c|}{$\begin{array}{l}\text { FY } 1996 \text { Unfunded } \\
\text { Full-Time Equivalents (FTEs) }\end{array}$} & \multicolumn{3}{|c|}{$\begin{array}{l}\text { FY } 1996 \text { Baseline } \\
\text { Full-Time Equivalents (FTEs) }\end{array}$} \\
\hline TYPE OF FTE & Exempt & Non-Exempt & Bargaining & Exempt & Non-Exempt & Bargaining & Exempt & Non-Exempt & Bargaining \\
\hline Organizational & 0 & $\mathbf{0}$ & & 0 & & & 0 & 0 & $\mathbf{0}$ \\
\hline Support & 23 & 2.5 & & 0 & & & 23 & 2.5 & $\mathbf{0}$ \\
\hline TOTALFTES & 23 & 2.5 & 0 & 0 & 0 & 0 & 23 & 2.5 & $\mathbf{0}$ \\
\hline COST ELEMENTS & \multicolumn{3}{|c|}{ FY 1996 Approved Funding Budget } & \multicolumn{3}{|c|}{ FY 1996 Unfunded Budget } & \multicolumn{3}{|c|}{ FY 1996 Baseline Budget } \\
\hline Labor - Regular & & & 1735.9 & & & & & & 1735.9 \\
\hline Labor - Overtime & & & 0 & & & & & & 0 \\
\hline 0 Total Labor & & & 1735.9 & & & 0 & & & 1735.9 \\
\hline 1 Materials & & & 413.1 & & & & & & 413.1 \\
\hline 2 Purchased Services & & & 2000 & & & & & & 2000 \\
\hline 3 Other Hanford & & & 0 & & & & & & 0 \\
\hline Subtotal Originated Costs & & & 4149 & & & 0 & & & 4149 \\
\hline 4 Site Services & & & 286.5 & & & & & & 286.5 \\
\hline 5 Internal Charges & & & 0 & & & & & & 0 \\
\hline 6 IRM Support & & & 66.9 & & & & & & 66.9 \\
\hline 7 Overheads & & & 343.6 & & & & & & 343.6 \\
\hline 8 Revenue & & & & & & & & & 0 \\
\hline TOTAL DOLLARS & & & 4846 & & & & & & 4846 \\
\hline
\end{tabular}
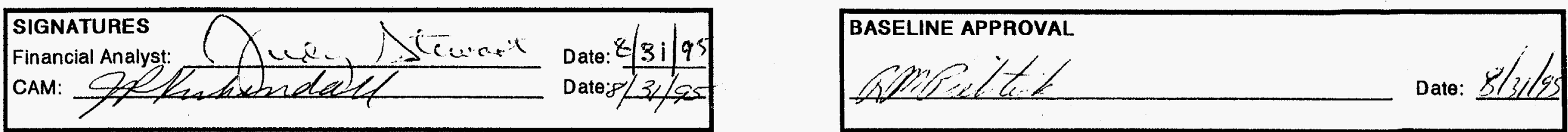


\section{Cost Account Narrative Summary}

This cost account provides for the funding of senior management-directed Reengineering teams to identify reengineering opportunities for implementation.

\section{Approved Funding Budget:}

Activity Detailed Description

Senior Management-Directed Reengineering

- includes $\$ 2 M$ CSC Index

25.5 FTES

Total Activity Cost

FY 1996

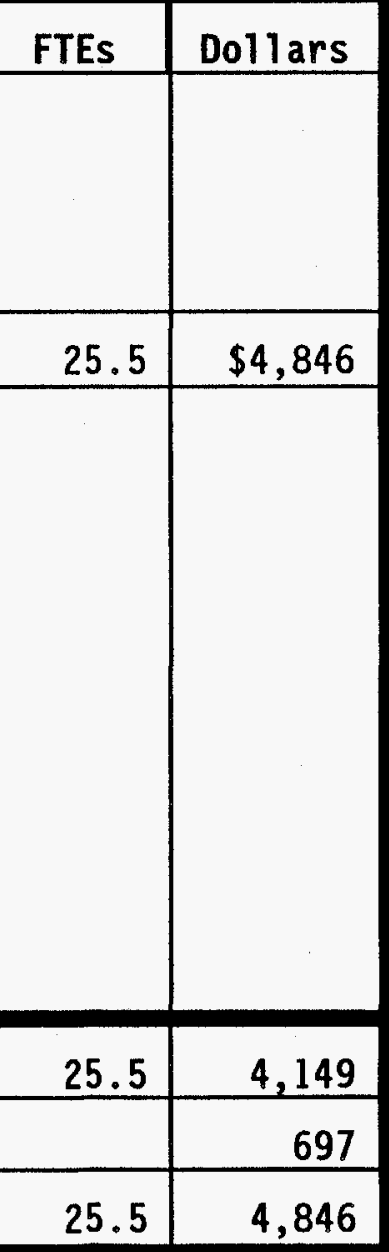

Total Approved Funding Originated Budget

Total Approved Funding Non-Originated Budget 
Part II Activity Detail

Unfunded Budget:

Activity Detailed Description

N/A
Cost Account No. IMDDCS

FY 1996

FTES Dollar

Total Activity Cost

Total Unfunded Originated Budget

Total Unfunded Non-Originated Budget

Total Unfunded Budget

Total Approved Funding \& Unfunded Budgets

4,846 


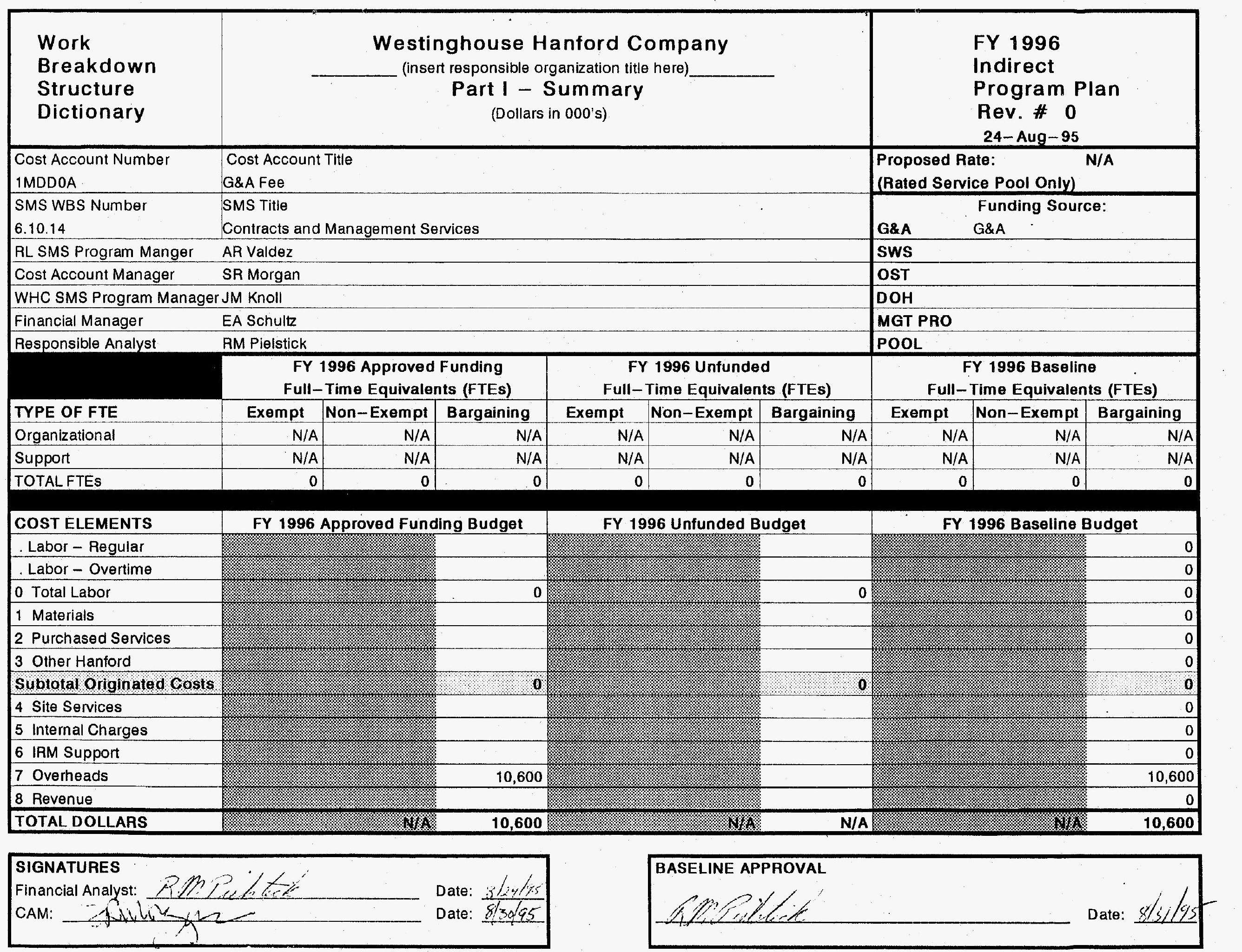




\section{COST ACCOUNT NARRATIVE SUMMARY}

This activity includes all of the fixed and award fees, plus the company-wide Performance Based Incentive fees.

APPROVED FUNDING BUDGET:

Activity Detailed Description

Fixed/Award and share of PBI fee

Activity Detailed Description

$$
\text { Total Activity Cost }
$$

Activity Detailed Description

Activity Detailed Description

$$
\text { Total Activity Cost }
$$

\section{Total Page}

Total Approved Funding Originated Budget

Total Approved Funding Non-Originated Budget

Total Approved Funding Budget 